\title{
Statistical Properties \& Different Methods Of Estimation Of A New Extended Weighted Frechet Distribution.
}

\author{
Mahdi Wahhab Neamah ${ }^{1}$, Nahla Hadi Abdul-Sahib ${ }^{2}$ \\ 1 Department of Statistics, Faculty of Administration and Economic, \\ Kerbala University, Iraq \\ Email: mehdi.wahab@uokerbala.edu.iq \\ 2 Department of Statistics, Faculty of Administration and Economic, \\ Kerbala University, Iraq \\ Email: nahlahadi@yahoo.com
}

Article History: Received: 11 January 2021; Accepted: 27 February 2021; Published online: 5 April 2021

\begin{abstract}
In this paper, we introduce a new distribution is called the extended weighted Frechet distribution, which we obtain by applying the Azzalini method and deduced some statistical properties such as mean, variance, coefficients of variation, coefficient of skewness, and coefficient of kurtosis. The parameters of the new distribution were estimated by the following estimation methods: Maximum Likelihood Method (MLE) and percentile method. We used the Monte Carlo simulation to compare the performances of the proposed estimators obtained through methods of estimation.
\end{abstract}

Keywords: Azalini's method, extended weighted Frechet distribution, Percentils method.

\section{Introduction}

Recently, the known distributions did not represent the data obtained from the real world, which became more complex than before, as the problems associated with that data made it more difficult in terms of representation and modeling. Therefore, the matter required all researchers in the field of statistics to develop and expand these distributions in order to infer through them the results that are unbiased and consistent. As well as to obtain application flexibility for these distributions in data representation and modeling.

The proposed distribution called the expanded weighted frechet distribution that we obtained from applying Azzalini's method is more flexible. Azzalini (1985) introduced the skew-normal distribution when adding a parameter to impart more flexibility to the normal distribution. Also, M. K. Shakhatrah (2012) introduced a new class of distributions called two-parameter exponential distribution (TWE). This new class of distributions generalizes the weighted exponential distribution (WE) proposed by Gupta and Kundu (2009). It turns out that (TWE) better than (WE) application of the two groups from real data. Abbas Mahdavi \& Leila Jabari (2017) proposed a new model using Azalini's method and demonstrated through the application that the proposed model is better and more flexible in application to real data. Abdulhakim A. Al-Babtain (2020) proposed a new model for Rayleigh distribution was called type I, the half logistic Rayleigh distribution, and the new model was applied to real data where the data was adopted (63) views from previous research by researchers (Kundu and Raqap, 2009) to clarify the importance and flexibility of the new Rayleigh distribution. In this paper, a new extended weighted frechet distribution has been proposed and studied. Frechet distribution was introduced by Maurice Frechet (French mathematician) (1878-1973). The frechet distribution has many applications in modeling and analyzing events such as earthquakes, floods and wind speeds, diseases, as well as in engineering fields to analyzing the statistical behavior of engineering material properties.

The frechet distribution is inverted Weibull distribution, also called the extreme value distribution. The probability density function (pdf) and the cumulative distribution function (CDF) for frechet distribution are (Kamran Abbas and Tang Yincai, 2012):

$$
\begin{aligned}
& f(x)=\frac{\lambda}{\theta}\left(\frac{\theta}{x}\right)^{\lambda+1} e^{-\left(\frac{\theta}{x}\right)^{\lambda}} \quad x>0 \\
& F(x)=e^{-\left(\frac{\theta}{x}\right)^{\lambda}} \quad x>0
\end{aligned}
$$

Where: $\lambda>0$ is the shape parameter of the distribution. , $\theta>0$ is the scale parameter distribution.

\section{Azalini's Method}

Mathematician Azzalini, A. (1985) introduced this method by inserting an additional parameter into the normal distribution to obtain a new distribution called the skew-normal distribution to achieve more flexibility in the normal distribution function as it is an extension of it. After that, many researchers inserted the shape parameter into 


\section{Statistical Properties \& Different Methods Of Estimation Of A New Extended Weighted Frechet} Distribution.

non-symmetric distributions such as the T-skew, Skew-Cauchy by (Gupta et al., 2002), and Skew-logistic distribution by (Nadarajah 2009).

The general formula for Azzalini's method is[9]:

where:

$$
f(x)=\frac{f(x) \cdot F(\alpha x)}{P\left(\alpha X_{1}>X_{2}\right)}
$$

$f(x)$ is the probability density function of frechet distribution.

$\mathrm{F}(\alpha \mathrm{x})$ is the cumulative distribution function after adding $\alpha$ parameter.

$\mathrm{X}_{1}, \mathrm{X}_{2}$ : is independent and identically random variables. Then, the conditional probability density function of $\mathrm{X}=\mathrm{X}_{1}$ given $\alpha \mathrm{X}_{1}>\mathrm{X}_{2}$.

Observe that :

$P\left(\alpha X_{1}>X_{2}\right)=\int_{0}^{\infty}\left[\int_{0}^{\alpha X_{1}} f_{2}\left(X_{2}\right) d X_{2}\right] f_{1}\left(X_{1}\right) d X_{1}$

$\mathrm{P}\left(\alpha \mathrm{X}_{1}>\mathrm{X}_{2}\right)=\int_{0}^{\infty}\left[\int_{0}^{\alpha \mathrm{X}_{1}} \frac{\lambda}{\theta}\left(\frac{\theta}{\mathrm{X}_{2}}\right)^{\lambda+1} \mathrm{e}^{-\left(\frac{\theta}{\mathrm{X}_{2}}\right)^{\lambda}} \mathrm{dX} \mathrm{X}_{2}\right] \frac{\lambda}{\theta}\left(\frac{\theta}{\mathrm{X}_{1}}\right)^{\lambda+1} \mathrm{e}^{-\left(\frac{\theta}{\mathrm{X}_{1}}\right)^{\lambda}} \mathrm{dX_{1 }}$

Then :

$\left.\mathrm{P}\left(\alpha \mathrm{X}_{1}>\mathrm{X}_{2}\right)=\frac{-1}{\frac{\alpha^{\lambda}+1}{\alpha^{\lambda}}} \mathrm{e}^{-\mathrm{t}^{\lambda}\left(\frac{\alpha^{\lambda}+1}{\alpha^{\lambda}}\right)}\right]_{0}^{\infty}$

$P\left(\alpha X_{1}>X_{2}\right)=\frac{\alpha^{\lambda}}{\alpha^{\lambda}+1}$

By (4) in the (3), we get the probability density function to the extended weighted frechet distribution new

(pdf).

$$
f(x, \lambda, \theta, \alpha)=\frac{\lambda}{\theta} \frac{\alpha^{\lambda}+1}{\alpha^{\lambda}}\left(\frac{\theta}{x}\right)^{\lambda+1} e^{-\left(\frac{\theta}{x}\right)^{\lambda}\left(\frac{\alpha^{\lambda}+1}{\alpha^{\lambda}}\right)}
$$

from eq. (5) we found the cumulative function to the new distribution by integration:

$\mathrm{F}(\mathrm{x}, \lambda, \theta, \alpha)=\int_{0}^{x} \frac{\lambda}{\theta} \frac{\alpha^{\lambda}+1}{\alpha^{\lambda}}\left(\frac{\theta}{\mathrm{x}}\right)^{\lambda+1} \mathrm{e}^{-\left(\frac{\theta}{\mathrm{x}}\right)^{\lambda}\left(\frac{\alpha^{\lambda}+1}{\alpha^{\lambda}}\right)} \mathrm{dx}$

$\mathrm{F}(\mathrm{x}, \lambda, \theta, \alpha)=\mathrm{e}^{-\left(\frac{\theta}{\mathrm{x}}\right)^{\lambda}\left(\frac{\alpha^{\lambda}+1}{\alpha^{\lambda}}\right)}$

\section{Characteristics of the new extended weighted Frechet Distribution}

\section{1: mean}

The mean can be obtained by finding The $\mathrm{r}^{\text {th }}$ moment about the origin when the value of $\mathrm{r}=1$ :

$$
\mu_{r}=E\left(x^{r}\right)=\int_{0}^{\infty} x^{r} f(x) d x
$$

By (1) in the (7), we get:

$\mu_{r}=E\left(x^{r}\right)=\int_{0}^{\infty} x^{r} \frac{\lambda}{\theta} \frac{\alpha^{\lambda}+1}{\alpha^{\lambda}}\left(\frac{\theta}{\mathrm{x}}\right)^{\lambda+1} \mathrm{e}^{-\left(\frac{\theta}{\mathrm{x}}\right)^{\lambda}\left(\frac{\alpha^{\lambda}+1}{\alpha^{\lambda}}\right)} d x$

The $\mathrm{r}^{\text {th }}$ moment about the origin of extended weighted Frechet distribution is:

$\mu_{r}=E\left(x^{r}\right)=\theta^{r} k^{\frac{r}{\lambda}} \Gamma\left(1-\frac{r}{\lambda}\right)$

At $\mathrm{r}=1$, we obtain the mean :

$\mu_{1}=E(x)=\theta k^{\frac{1}{\lambda}} \Gamma\left(1-\frac{1}{\lambda}\right)$

\section{2: Variance}

The Variance can be obtained by finding The $\mathrm{r}^{\text {th }}$ moment about the mean when the value of $\mathrm{r}=2$ :

$$
E(x-\mu)^{r}=\int_{0}^{\infty}(x-\mu)^{r} f(x) d x
$$

By (1) in the (9), we get:

$E(x-\mu)^{r}=\int_{0}^{\infty}(x-\mu)^{r} \frac{\lambda}{\theta} \frac{\alpha^{\lambda}+1}{\alpha^{\lambda}}\left(\frac{\theta}{x}\right)^{\lambda+1} e^{-\left(\frac{\theta}{x}\right)^{\lambda}\left(\frac{\alpha^{\lambda}+1}{\alpha^{\lambda}}\right)} d x$ 
The $\mathrm{r}^{\text {th }}$ moment about the mean of extended weighted Frechet distribution is:

$E(x-\mu)^{r}=\sum_{j=0}^{r}\left(\begin{array}{l}r \\ j\end{array}\right) \theta^{j}\left(k^{\frac{j}{\lambda}}\right)(-\mu)^{r-j} \Gamma\left(1-\frac{j}{\lambda}\right)$

At $r=2$, we obtain the variance:

$\mathrm{E}(\mathrm{x}-\mu)^{2}=\sum_{\mathrm{j}=0}^{2}\left(\begin{array}{l}2 \\ \mathrm{j}\end{array}\right) \theta^{\mathrm{j}}\left(\mathrm{k}^{\frac{\mathrm{j}}{\lambda}}\right)(-\mu)^{2-\mathrm{j}} \Gamma\left(1-\frac{1}{\lambda}\right)=\operatorname{var}(\mathrm{x})$

\section{3: Coefficients of Variation}

$C . V=\frac{\sigma}{\mu} \times 100$

The mathematical formula for the coefficient of variation is:

\section{4: Coefficient of Skewness}

$$
C . V=\frac{\sqrt{\Gamma\left(1-\frac{2}{\lambda}\right)-\left(\Gamma\left(1-\frac{1}{\lambda}\right)\right)^{2}}}{\Gamma\left(1-\frac{1}{\lambda}\right)} \times 100
$$

The mathematical formula for the Coefficient of Skewness is:

C.S $=\frac{E(x-\mu)^{3}}{\sigma^{3}}$

$C . S=\frac{-\theta^{3} \mathrm{k}^{\frac{3}{\lambda}} \Gamma\left(1-\frac{1}{\lambda}\right)^{3}+3 \theta k^{1 / \lambda} \theta \mathrm{k}^{\frac{1}{\lambda}} \Gamma\left(1-\frac{1}{\lambda}\right)^{2}-3 \theta^{3} k^{3 / \lambda} \Gamma\left(1-\frac{1}{\lambda}\right) \Gamma\left(1-\frac{2}{\lambda}\right)+\theta^{3} k^{3 / \lambda} \Gamma\left(1-\frac{3}{\lambda}\right)}{\theta^{3} \mathrm{k}^{3 / \lambda}\left(\Gamma\left(1-\frac{2}{\lambda}\right)-\Gamma\left(1-\frac{1}{\lambda}\right)^{2}\right)^{3 / 2}}$

\section{5: Coefficient of Kurtosis}

The mathematical formula for the Coefficient of Kurtosis is:

$$
K . S=\frac{\frac{E(x-\mu)^{4}}{\sigma^{4}}}{\left(-3 \Gamma\left(1-\frac{1}{\lambda}\right)^{4}+6 \Gamma\left(1-\frac{1}{\lambda}\right)^{2} \Gamma\left(1-\frac{2}{\lambda}\right)-4 \Gamma\left(1-\frac{1}{\lambda}\right) \Gamma\left(1-\frac{3}{\lambda}\right)+\Gamma\left(1-\frac{4}{\lambda}\right)\right)}
$$

\section{Estimation}

\section{1: Maximum Likelihood Method (MLE)}

If $\mathrm{x}_{1}, \mathrm{x}_{2} \ldots \mathrm{x}_{\mathrm{n}}$ are random variables distributed in the extended weighted Freight distribution, then:

$$
\begin{aligned}
& \mathrm{f}(\mathrm{x}, \lambda, \theta, \alpha)=\frac{\lambda}{\theta}\left(1+\alpha^{-\lambda}\right)\left(\frac{\theta}{\mathrm{x}}\right)_{n}^{\lambda+1} \mathrm{e}^{-\left(\frac{\theta}{\mathrm{x}}\right)^{\lambda}\left(1+\alpha^{-\lambda}\right)} \\
& L\left(x_{1}, x_{2} \ldots \ldots \ldots x_{n}, \lambda, \theta, \alpha\right)=\prod_{i=1}^{n} f\left(x_{i}, \lambda, \theta, \alpha\right) \\
& L(x, \lambda, \theta, \alpha)=\frac{\lambda^{n}}{\theta}\left(1+\alpha^{-\lambda}\right)^{n} \prod_{i=1}^{n}\left(\frac{\theta}{\mathrm{x}}\right)^{\lambda+1} \mathrm{e}^{-\left(1+\alpha^{-\lambda}\right) \sum_{i=1}^{n}\left(\frac{\theta}{\mathrm{x}}\right)^{\lambda}} \\
& \ln L=n \ln \lambda-n \ln \theta+n \ln \left(1+\alpha^{-\lambda}\right)+(\lambda+1) \sum \ln \left(\frac{\theta}{x_{i}}\right)-\left(1+\alpha^{-\lambda}\right) \sum\left(\frac{\theta}{x_{i}}\right)^{\lambda} \\
& \frac{\partial \ln L}{\partial \lambda}=\frac{n}{\lambda}-\frac{n \alpha^{-\lambda} \ln (\alpha)}{\left(1+\alpha^{-\lambda}\right)}+\sum \ln \left(\frac{\theta}{x_{i}}\right)-\sum\left(\frac{\theta}{x_{i}}\right)^{\lambda} \ln \left(\frac{\theta}{x_{i}}\right)-\alpha^{-\lambda} \sum\left(\frac{\theta}{x_{i}}\right)^{\lambda} \ln \left(\frac{\theta}{x_{i}}\right)+\sum\left(\frac{\theta}{x_{i}}\right)^{\lambda} \alpha^{-\lambda} \ln \alpha
\end{aligned}
$$

To estimate the parameter $(\theta)$ :

$$
\frac{\partial \operatorname{lnL}}{\partial \theta}=-\frac{n}{\theta}+\frac{\lambda \sum \frac{1}{x_{i}}}{\sum\left(\frac{\theta}{x_{i}}\right)}+\frac{\sum \frac{1}{x_{i}}}{\sum\left(\frac{\theta}{x_{i}}\right)}-\alpha \sum\left(\frac{\theta}{x_{i}}\right)^{\lambda-1}\left(\frac{1}{x_{i}}\right)-\lambda \alpha^{-\lambda} \sum\left(\frac{\theta}{x_{i}}\right)^{\lambda-1}\left(\frac{1}{x_{i}}\right)
$$

To estimate the parameter $(\alpha)$ : 
Statistical Properties \& Different Methods Of Estimation Of A New Extended Weighted Frechet Distribution.

$$
\begin{aligned}
& \frac{\partial \operatorname{lnL}}{\partial \alpha}=\frac{n(-\lambda) \alpha^{-(\lambda+1)}}{\left(1+\alpha^{-\lambda}\right)}+\lambda \alpha^{-(\lambda+1)} \sum\left(\frac{\theta}{x_{i}}\right)^{\lambda} \\
& \text { The MLE } \hat{\lambda}, \hat{\theta} \text { and } \hat{\alpha} \text { Can be obtained by solving the likelihood eqs. } \\
& \qquad\left.\frac{\partial \ln L}{\partial \lambda}\right|_{\lambda=\hat{\lambda}}=0 \quad,\left.\quad \frac{\partial \ln L}{\partial \theta}\right|_{\theta=\hat{\theta}}=0 \quad \text { and }\left.\frac{\partial \ln L}{\partial \theta}\right|_{\alpha=\widehat{\alpha}}=0
\end{aligned}
$$

Clearly, it is difficult to solve the equations (10), (11), and (12) therefore applying Newton-Raphson's method.

\section{2: Maximum product of spacing estimator method}

$$
\begin{aligned}
& F(x)=e^{-\left(\frac{\theta}{x}\right)^{\lambda}\left(\frac{\alpha^{\lambda}+1}{\alpha^{\lambda}}\right)} \\
& F(x)=e^{-k\left(\frac{\theta}{x}\right)^{\lambda}} \\
& G=\left(\prod_{i=1}^{n+1} D_{i}\right)^{\frac{1}{n+1}} \\
& D_{i}=\left\{\begin{array}{c}
D_{1}=F\left(x_{1}\right) \\
D_{i}=F\left(x_{i}\right)-F\left(x_{i-1}\right)=F\left(x_{(2: m)}\right) ; \quad i=2, \ldots, m \\
D_{m+1}=1-F\left(x_{m}\right)
\end{array}\right. \\
& \ln G=\frac{1}{n+1}\left[\left(-\left(\frac{\alpha^{\lambda}+1}{\alpha^{\lambda}}\right)\left(\frac{\theta}{x_{1}}\right)^{\lambda}\right)+\sum_{i=2}^{n} \ln \left(e^{-\left(\frac{\alpha^{\lambda}+1}{\alpha^{\lambda}}\right)\left(\frac{\theta}{x_{i}}\right)^{\lambda}}-e^{-\left(\frac{\alpha^{\lambda}+1}{\alpha^{\lambda}}\right)\left(\frac{\theta}{x_{i-1}}\right)^{\lambda}}\right)+\ln \left(1-e^{-\left(\frac{\alpha^{\lambda}+1}{\alpha^{\lambda}}\right)\left(\frac{\theta}{x_{n}}\right)^{\lambda}}\right)\right]
\end{aligned}
$$

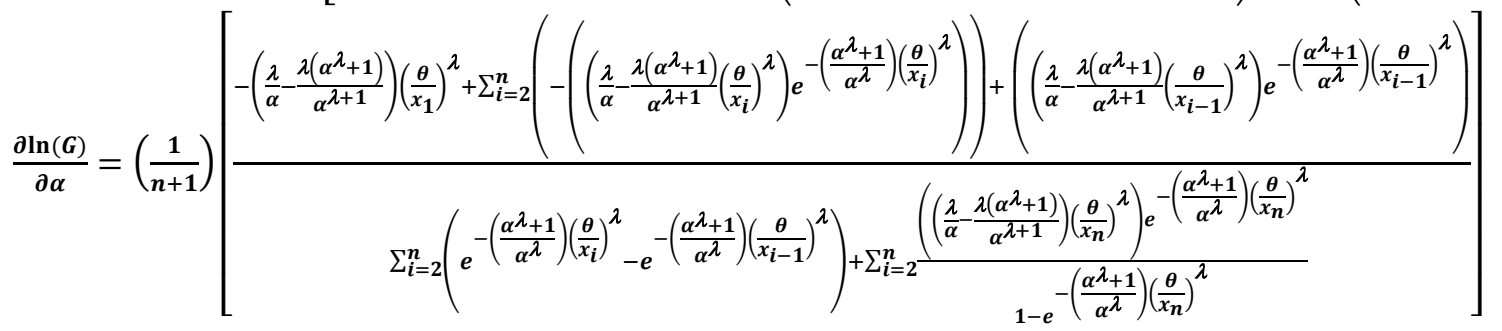

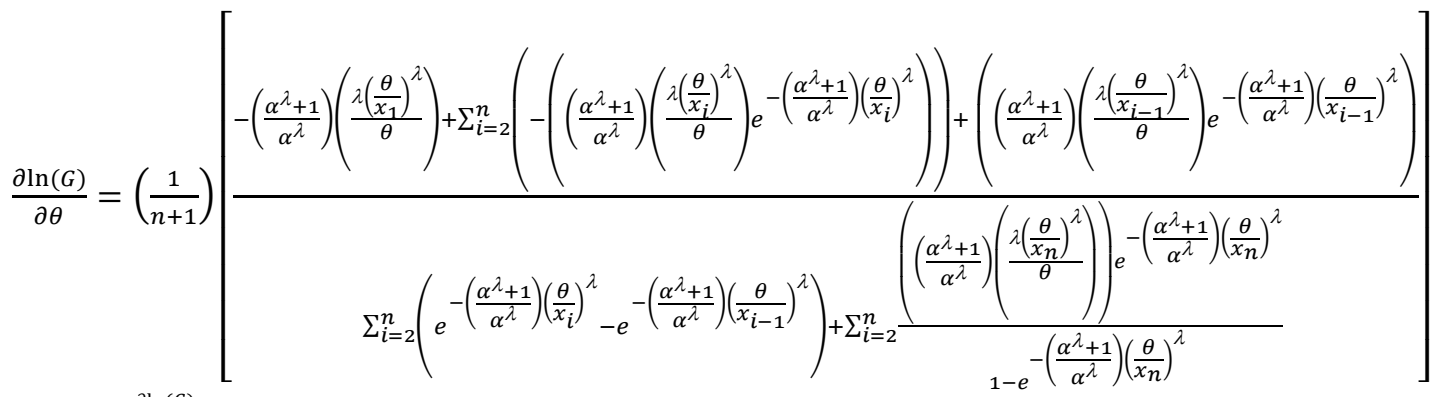

$$
\frac{\partial \ln (G)}{\partial \lambda}
$$

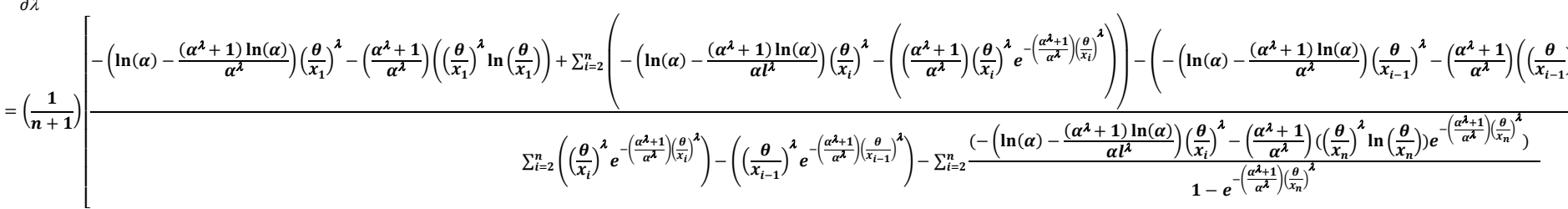

The MLE $\hat{\lambda}, \hat{\theta}$ and $\hat{\alpha}$ can be obtained by solving the likelihood eqs.

$$
\left.\frac{\partial \ln G}{\partial \lambda}\right|_{\lambda=\hat{\lambda}}=0 \quad,\left.\quad \frac{\partial \ln G}{\partial \theta}\right|_{\theta=\hat{\theta}}=0 \quad \text { and }\left.\frac{\partial \ln G}{\partial \theta}\right|_{\alpha=\widehat{\alpha}}=0
$$

Clearly, it is difficult to solve the equations (13), (14), and (15) therefore applying Newton-Raphson's method.

\section{3: Method of Cramer-Von Mises Minimum} estimators

The CVME, as a type of minimum distance estimator, has less bias than the other minimum distance 


$$
\begin{gathered}
C(\lambda, \alpha, \theta)=\frac{1}{12 n}+\sum_{i=1}^{n}\left[F\left(x_{i}, \lambda, \alpha, \theta\right)-\frac{2 i-1}{2 n}\right]^{2} \\
C(\lambda, \alpha, \theta)=\frac{1}{12 n}+\sum_{i=1}^{n}\left[e^{-\left(\frac{\theta}{x}\right)^{\lambda}\left(\frac{\alpha^{\lambda}+1}{\alpha^{\lambda}}\right)}-\frac{2 i-1}{2 n}\right]^{2} \\
\frac{\partial C(\lambda, \alpha, \theta)}{\partial \lambda}=2 \sum_{i=1}^{n}\left[e^{-\left(\frac{\theta}{x}\right)^{\lambda}\left(\frac{\alpha^{\lambda}+1}{\alpha^{\lambda}}\right)}\right. \\
\left.-\frac{2 i-1}{2 n}\right]\left[e ^ { - ( \frac { \theta } { x } ) ^ { \lambda } ( \frac { \alpha ^ { \lambda } + 1 } { \alpha ^ { \lambda } } ) } \left[-\left(\left(\frac{\theta}{x}\right)^{\lambda}\left(\frac{\alpha^{\lambda}\left(\frac{\alpha^{\lambda}+1}{\alpha^{\lambda}}\right) \ln \alpha}{\alpha^{2 \lambda}}\right)-\left(\alpha^{\lambda}+1\right) \alpha^{\lambda} \ln (\alpha)\right)\right.\right. \\
\left.+\left(\frac{\alpha^{\lambda}+1}{\alpha^{\lambda}}\right)\left(\frac{\theta}{x}\right)^{\lambda} \ln \left(\frac{\theta}{x}\right)\right)
\end{gathered}
$$

And

$$
\frac{\partial C(\lambda, \alpha, \theta)}{\partial \theta}=2 \sum_{i=1}^{n}\left[e^{-\left(\frac{\theta}{x}\right)^{\lambda}\left(\frac{\alpha^{\lambda}+1}{\alpha^{\lambda}}\right)}-\frac{2 i-1}{2 n}\right]\left[e^{-\left(\frac{\theta}{x}\right)^{\lambda}\left(\frac{\alpha^{\lambda}+1}{\alpha^{\lambda}}\right)} \lambda\left(\frac{\alpha^{\lambda}+1}{\alpha^{\lambda}}\right)\left(\frac{\theta}{x}\right)^{\lambda-1}\left(\frac{1}{x}\right)\right]=0
$$

And

$$
\frac{\partial C(\lambda, \alpha, \theta)}{\partial \alpha}==-2 \sum_{i=1}^{n}\left[e^{-\left(\frac{\theta}{x}\right)^{\lambda}\left(\frac{\alpha^{\lambda}+1}{\alpha^{\lambda}}\right)}-\frac{2 i-1}{2 n}\right]\left[e^{-\left(\frac{\theta}{x}\right)^{\lambda}\left(\frac{\alpha^{\lambda}+1}{\alpha^{\lambda}}\right)}\left(\left(\frac{\theta}{x}\right)^{\lambda}\left(\frac{\lambda}{\alpha}-\frac{\left(\alpha^{\lambda}+1\right) \lambda}{\alpha^{\lambda} \alpha}\right)\right)\right]
$$

The MLE $\hat{\lambda}, \hat{\theta}$ and $\hat{\alpha}$ can be obtained by solving the likelihood eqs.

$$
\left.\frac{\partial \ln C}{\partial \lambda}\right|_{\lambda=\hat{\lambda}}=0 \quad,\left.\quad \frac{\partial \ln C}{\partial \theta}\right|_{\theta=\hat{\theta}}=0 \quad \text { and }\left.\frac{\partial \ln C}{\partial \theta}\right|_{\alpha=\widehat{\alpha}}=0
$$

\begin{tabular}{|c|c|c|c|c|c|c|c|c|c|c|c|}
\hline \multicolumn{3}{|c|}{$\begin{array}{c}\text { Estimated } \\
\text { parameters }\end{array}$} & $\hat{\lambda}$ & $\widehat{\boldsymbol{\theta}}$ & $\widehat{\alpha}$ & $\hat{\lambda}$ & $\widehat{\boldsymbol{\theta}}$ & $\widehat{\alpha}$ & $\hat{\lambda}$ & $\widehat{\boldsymbol{\theta}}$ & $\widehat{\alpha}$ \\
\hline \multirow{2}{*}{$\mathbf{N}$} & \multirow[b]{2}{*}{ ti } & \multirow{2}{*}{ Real } & $\begin{array}{c}1.7 \\
1 \\
\end{array}$ & $\begin{array}{c}2.7 \\
7 \\
\end{array}$ & $\begin{array}{c}7.2 \\
9 \\
\end{array}$ & $\begin{array}{c}2.2 \\
7 \\
\end{array}$ & $\begin{array}{c}3.9 \\
1\end{array}$ & \begin{tabular}{|c}
3.5 \\
2 \\
\end{tabular} & \begin{tabular}{|c|}
2.0 \\
0 \\
\end{tabular} & $\begin{array}{c}4.0 \\
0\end{array}$ & 4.00 \\
\hline & & & Mle & \multicolumn{2}{|c|}{ MSE } & MPS & \multicolumn{2}{|c|}{ MSE } & $\begin{array}{l}\text { CV } \\
\text { MD } \\
\end{array}$ & \multicolumn{2}{|c|}{ MSE } \\
\hline \multirow{6}{*}{35} & $\begin{array}{c}13.298 \\
4\end{array}$ & 0.1985 & 0.2578 & & $\begin{array}{l}.0302 \\
\{3\}\end{array}$ & $\begin{array}{c}0.226 \\
7\end{array}$ & & $\overline{0008^{12}}$ & $\begin{array}{c}0.19 \\
86\end{array}$ & \multicolumn{2}{|c|}{$\begin{array}{c}0.000003 \\
\{1\}\end{array}$} \\
\hline & $\begin{array}{c}12.885 \\
3\end{array}$ & 0.1984 & $0.257^{7}$ & & $\begin{array}{l}.0281 \\
\{3\}\end{array}$ & $\begin{array}{c}0.226 \\
7\end{array}$ & & $008^{\{2}$ & $\begin{array}{c}0.19 \\
86\end{array}$ & \multicolumn{2}{|c|}{$\begin{array}{c}0.000003 \\
\{1\}\end{array}$} \\
\hline & $\begin{array}{c}12.526 \\
0\end{array}$ & 0.1884 & 0.256 & & $\begin{array}{l}.0228 \\
\{3\}\end{array}$ & $\begin{array}{c}0.208 \\
6\end{array}$ & & $006^{\{2}$ & $\begin{array}{c}0.18 \\
85\end{array}$ & \multicolumn{2}{|c|}{$\begin{array}{c}0.000003 \\
\{1\}\end{array}$} \\
\hline & $\begin{array}{c}12.400 \\
7\end{array}$ & 0.1844 & 0.240 & & $\begin{array}{l}.0074 \\
\{3\}\end{array}$ & $\begin{array}{c}0.208 \\
3\end{array}$ & & $005^{12}$ & $\begin{array}{c}0.18 \\
45\end{array}$ & \multicolumn{2}{|c|}{$\begin{array}{c}0.000003 \\
\{1\}\end{array}$} \\
\hline & $\begin{array}{c}12.379 \\
6\end{array}$ & 0.1833 & 0.240 & & $\begin{array}{l}.0073 \\
\{3\}\end{array}$ & $\begin{array}{c}0.202 \\
4\end{array}$ & & $004^{\{2}$ & $\begin{array}{c}0.18 \\
34\end{array}$ & \multicolumn{2}{|c|}{$\begin{array}{c}0.000002 \\
\{1\}\end{array}$} \\
\hline & 11.140 & 0.1787 & 0.229 & & .0032 & 0.199 & & $003^{12}$ & 0.17 & \multicolumn{2}{|c|}{0.000002} \\
\hline
\end{tabular}

Clearly, it is difficult to solve the equations (), (), therefore applying Newton-Raphson's method.

\section{Simulation Study}

We ran a simulation to compare the behavior of the estimates with respect to mean squares of error (MSEs) using the rank method, as this method is based on selecting the lowest order for the sum of the total and partial ranks of all estimation methods for a set of imposed parameter values $(\hat{\lambda}, \hat{\theta}, \hat{\alpha})$. The results were as shown in Table (1).

Table (1): Simulation results for $(\lambda=2, \theta=4, \alpha=4)$ 
Statistical Properties \& Different Methods Of Estimation Of A New Extended Weighted Frechet Distribution.

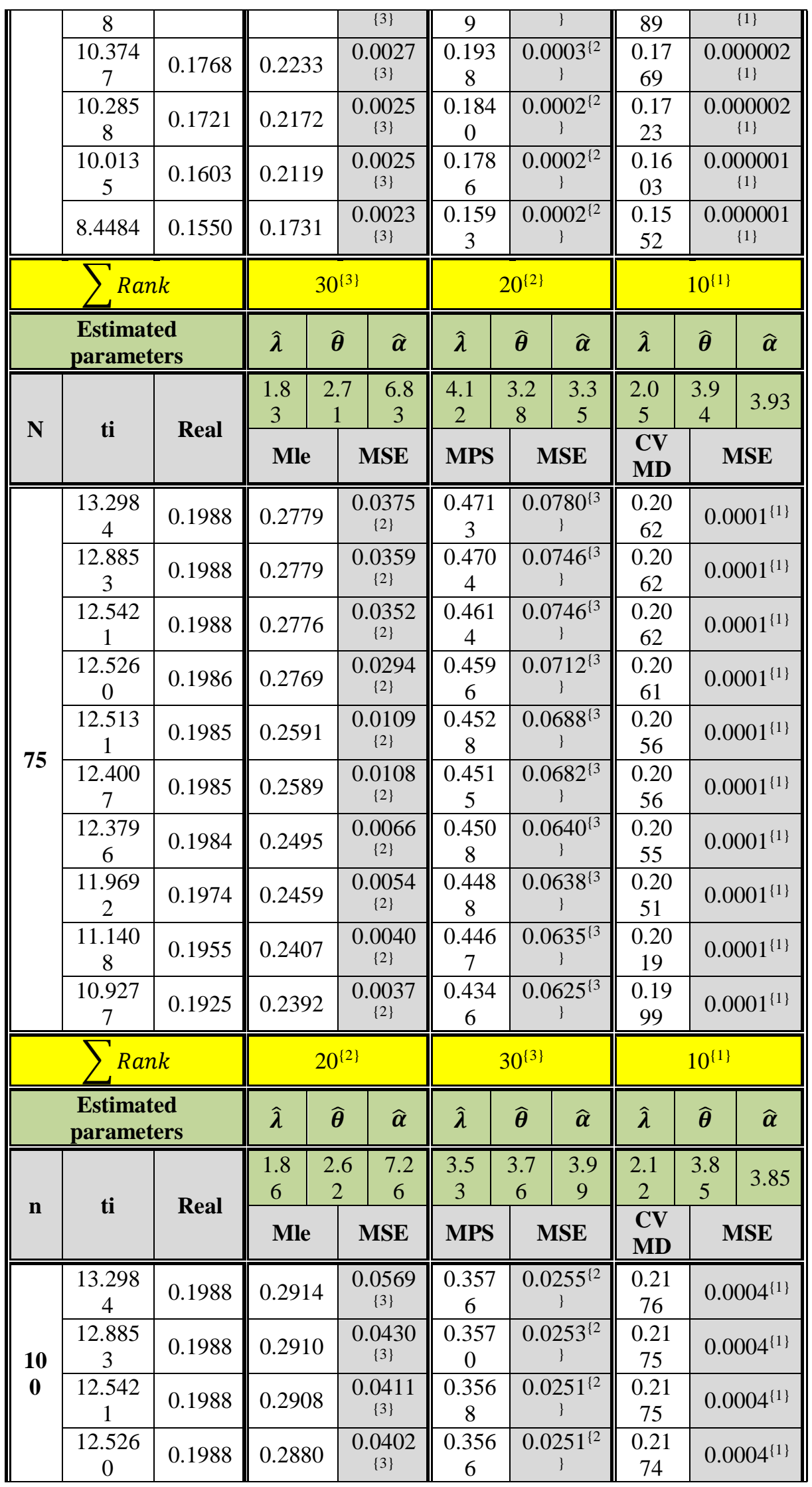


Mahdi Wahhab Neamah ${ }^{1}$, Nahla Hadi Abdul-Sahib ${ }^{2}$

\begin{tabular}{|c|c|c|c|c|c|c|c|}
\hline $\begin{array}{c}12.513 \\
1 \\
\end{array}$ & 0.1986 & 0.2851 & $\begin{array}{c}0.0334 \\
\{3\}\end{array}$ & $\begin{array}{c}0.354 \\
4\end{array}$ & $\begin{array}{c}0.0250^{\{2} \\
\}\end{array}$ & $\begin{array}{c}0.21 \\
74 \\
\end{array}$ & $0.0004^{\{1\}}$ \\
\hline $\begin{array}{c}12.400 \\
7\end{array}$ & 0.1985 & 0.2813 & $\begin{array}{c}0.0289 \\
\{3\}\end{array}$ & $\begin{array}{c}0.351 \\
8\end{array}$ & $\begin{array}{c}0.0240^{\{2} \\
\}\end{array}$ & $\begin{array}{c}0.21 \\
70\end{array}$ & $0.0004^{\{1\}}$ \\
\hline $\begin{array}{c}12.379 \\
6\end{array}$ & 0.1985 & 0.2751 & $\begin{array}{c}0.0188 \\
\{2\}\end{array}$ & $\begin{array}{c}0.350 \\
8\end{array}$ & $\begin{array}{c}0.0238^{\{3} \\
\}\end{array}$ & $\begin{array}{c}0.21 \\
63\end{array}$ & $0.0004^{\{1\}}$ \\
\hline $\begin{array}{c}11.969 \\
2\end{array}$ & 0.1984 & 0.2644 & $\begin{array}{c}0.0120 \\
\{2\}\end{array}$ & $\begin{array}{c}0.350 \\
5 \\
\end{array}$ & $\begin{array}{c}0.0234^{\{3} \\
\}\end{array}$ & $\begin{array}{c}0.21 \\
62 \\
\end{array}$ & $0.0004^{\{1\}}$ \\
\hline $\begin{array}{c}11.141 \\
6\end{array}$ & 0.1981 & 0.2642 & $\begin{array}{c}0.0119 \\
\{2\}\end{array}$ & $\begin{array}{c}0.349 \\
1\end{array}$ & $\begin{array}{c}0.0231^{\{3} \\
\end{array}$ & $\begin{array}{c}0.21 \\
60\end{array}$ & $0.0004^{\{1\}}$ \\
\hline $\begin{array}{c}11.140 \\
8 \\
\end{array}$ & 0.1974 & 0.2531 & $\begin{array}{c}0.0072 \\
\{2\}\end{array}$ & $\begin{array}{c}0.348 \\
0 \\
\end{array}$ & $\begin{array}{c}0.0230^{\{3} \\
\}\end{array}$ & $\begin{array}{c}0.21 \\
54 \\
\end{array}$ & $0.0004^{\{1\}}$ \\
\hline$\sum R a$ & & & & & $4^{\{2\}}$ & & $0^{\{1\}}$ \\
\hline Over ral & & & & & 10 & & 3 \\
\hline
\end{tabular}

Table (2): Simulation results for $(\lambda=3.5, \theta=4, \alpha=3.5)$

\begin{tabular}{|c|c|c|c|c|c|c|c|c|c|c|c|}
\hline \multicolumn{3}{|c|}{$\begin{array}{l}\text { Estimated } \\
\text { parameters }\end{array}$} & $\hat{\lambda}$ & $\widehat{\boldsymbol{\theta}}$ & $\widehat{\boldsymbol{\alpha}}$ & $\hat{\lambda}$ & $\widehat{\boldsymbol{\theta}}$ & $\widehat{\alpha}$ & $\hat{\lambda}$ & $\widehat{\boldsymbol{\theta}}$ & $\widehat{\boldsymbol{\alpha}}$ \\
\hline \multirow{2}{*}{$\mathbf{N}$} & \multirow{2}{*}{ Ti } & \multirow{2}{*}{ Real } & $\begin{array}{c}2.1 \\
9\end{array}$ & $\begin{array}{c}2.6 \\
5\end{array}$ & $\begin{array}{c}6.2 \\
8\end{array}$ & $\begin{array}{c}3.6 \\
8\end{array}$ & $\begin{array}{c}5.3 \\
0\end{array}$ & $\begin{array}{c}1.5 \\
2\end{array}$ & $\begin{array}{c}3.1 \\
1\end{array}$ & $\begin{array}{c}4.1 \\
5\end{array}$ & 3.83 \\
\hline & & & Mle & \multicolumn{2}{|c|}{ MSE } & MPS & \multicolumn{2}{|c|}{ MSE } & $\begin{array}{l}\text { CV } \\
\text { MD }\end{array}$ & \multicolumn{2}{|c|}{ MSE } \\
\hline \multirow{10}{*}{35} & 9.8791 & 0.3329 & $\begin{array}{c}0.250 \\
3\end{array}$ & \multicolumn{2}{|c|}{$\begin{array}{c}0.0865^{\{} \\
3\}\end{array}$} & $\begin{array}{c}0.286 \\
0\end{array}$ & \multicolumn{2}{|c|}{$\begin{array}{c}0.0296^{\{2} \\
\}\end{array}$} & $\begin{array}{c}0.28 \\
60\end{array}$ & \multicolumn{2}{|c|}{$0.0022^{\{1\}}$} \\
\hline & 9.5171 & 0.3329 & $\begin{array}{c}0.249 \\
4\end{array}$ & \multicolumn{2}{|c|}{$\begin{array}{c}0.0836 \\
3\}\end{array}$} & $\begin{array}{c}0.282 \\
6\end{array}$ & \multicolumn{2}{|c|}{$\begin{array}{c}0.0291^{12} \\
\end{array}$} & $\begin{array}{c}0.28 \\
59\end{array}$ & \multicolumn{2}{|c|}{$0.0022^{\{1\}}$} \\
\hline & 9.2221 & 0.3168 & $\begin{array}{c}0.248 \\
3\end{array}$ & \multicolumn{2}{|c|}{$\begin{array}{c}0.0803^{\{} \\
3\}\end{array}$} & $\begin{array}{c}0.274 \\
3\end{array}$ & \multicolumn{2}{|c|}{$0.0261^{\{2}$} & $\begin{array}{c}0.28 \\
07\end{array}$ & \multicolumn{2}{|c|}{$0.0022^{\{1\}}$} \\
\hline & 9.1230 & 0.3078 & $\begin{array}{c}0.248 \\
2\end{array}$ & \multicolumn{2}{|c|}{$\begin{array}{c}0.0799 \\
3\}\end{array}$} & $\begin{array}{c}0.243 \\
6\end{array}$ & \multicolumn{2}{|c|}{$\begin{array}{c}0.0246^{\{2} \\
\}\end{array}$} & $\begin{array}{c}0.27 \\
54\end{array}$ & \multicolumn{2}{|c|}{$0.0021^{\{1\}}$} \\
\hline & 9.1065 & 0.3069 & $\begin{array}{c}0.243 \\
8\end{array}$ & \multicolumn{2}{|c|}{$\begin{array}{c}0.0792\{ \\
3\}\end{array}$} & $\begin{array}{c}0.243 \\
4\end{array}$ & & $44^{\{2}$ & $\begin{array}{c}0.26 \\
00\end{array}$ & & $19^{\{1\}}$ \\
\hline & 8.2114 & 0.2993 & $\begin{array}{c}0.241 \\
9\end{array}$ & & $739^{1}$ & 0.229 & & $38^{\{2}$ & 0.25 & & $17^{\{1\}}$ \\
\hline & 7.7136 & 0.2888 & $\begin{array}{c}0.236 \\
9\end{array}$ & & $600^{1}$ & $\begin{array}{c}0.223 \\
3\end{array}$ & & $17^{\{2}$ & $\begin{array}{c}0.25 \\
35\end{array}$ & & $15^{\{1\}}$ \\
\hline & 7.6579 & 0.2834 & $\begin{array}{c}0.220 \\
2\end{array}$ & & $598^{1}$ & $\begin{array}{c}0.217 \\
0\end{array}$ & & $99^{\{2}$ & $\begin{array}{c}0.24 \\
49\end{array}$ & & $13^{\{1\}}$ \\
\hline & 7.4895 & 0.2770 & $\begin{array}{c}0.215 \\
4\end{array}$ & & $408^{\prime}$ & $\begin{array}{c}0.211 \\
7\end{array}$ & & $46^{\{2}$ & $\begin{array}{c}0.23 \\
92\end{array}$ & & $12^{\{1\}}$ \\
\hline & 6.5717 & 0.2547 & $\begin{array}{c}0.210 \\
4\end{array}$ & & $302^{\prime}$ & $\begin{array}{c}0.176 \\
6\end{array}$ & & $27^{\{2}$ & $\begin{array}{c}0.23 \\
54\end{array}$ & & $10^{\{1\}}$ \\
\hline & ${ }_{R}$ & & & $0^{\{3\}}$ & & & $20^{\{2\}}$ & & & $10^{\{1}$ & \\
\hline & $\begin{array}{l}\text { Estima } \\
\text { parame }\end{array}$ & & $\hat{\lambda}$ & $\widehat{\boldsymbol{\theta}}$ & $\widehat{\alpha}$ & $\hat{\lambda}$ & $\widehat{\boldsymbol{\theta}}$ & $\widehat{\alpha}$ & $\hat{\lambda}$ & $\widehat{\boldsymbol{\theta}}$ & $\widehat{\alpha}$ \\
\hline $\mathrm{I}$ & TT: & D & $\begin{array}{c}2.3 \\
3\end{array}$ & $\begin{array}{c}2.6 \\
0\end{array}$ & $\begin{array}{c}6.2 \\
7\end{array}$ & $\begin{array}{c}3.5 \\
4\end{array}$ & $\begin{array}{c}4.1 \\
7\end{array}$ & $\begin{array}{c}3.7 \\
1\end{array}$ & $\begin{array}{c}3.2 \\
2\end{array}$ & $\begin{array}{c}4.1 \\
0\end{array}$ & 3.74 \\
\hline IV & 11 & Kea & Mle & & SE & MPS & & SE & $\begin{array}{l}\text { CV } \\
\text { MD }\end{array}$ & & SE \\
\hline 75 & 9.8791 & 0.3329 & $\begin{array}{c}0.301 \\
6\end{array}$ & & $299^{\prime}$ & $\begin{array}{c}0.322 \\
3\end{array}$ & & $12^{\{1}$ & $\begin{array}{c}0.30 \\
01\end{array}$ & & $27^{\{2\}}$ \\
\hline
\end{tabular}


Statistical Properties \& Different Methods Of Estimation Of A New Extended Weighted Frechet Distribution.

\begin{tabular}{|c|c|c|c|c|c|c|c|c|c|}
\hline & 9.5171 & 0.3329 & $\begin{array}{c}0.298 \\
9\end{array}$ & $\begin{array}{c}0.0299\{ \\
3\}\end{array}$ & $\begin{array}{c}0.321 \\
4\end{array}$ & $0.0012^{\{1}$ & $\begin{array}{c}0.29 \\
97\end{array}$ & \multicolumn{2}{|c|}{$0.0027^{\{2\}}$} \\
\hline & 9.2350 & 0.3329 & $\begin{array}{c}0.297 \\
6\end{array}$ & $\begin{array}{c}0.0296^{1} \\
3\}\end{array}$ & $\begin{array}{c}0.319 \\
7\end{array}$ & $0.0012^{\{1}$ & $\begin{array}{c}0.29 \\
96\end{array}$ & \multicolumn{2}{|c|}{$0.0026^{\{2\}}$} \\
\hline & 9.2221 & 0.3317 & $\begin{array}{c}0.288 \\
0\end{array}$ & $\begin{array}{c}0.0291^{\{} \\
3\}\end{array}$ & $\begin{array}{c}0.318 \\
3\end{array}$ & $0.0012^{\{1}$ & $\begin{array}{c}0.29 \\
95\end{array}$ & \multicolumn{2}{|c|}{$0.0024^{\{2\}}$} \\
\hline & 9.2119 & 0.3315 & $\begin{array}{c}0.252 \\
8\end{array}$ & $\begin{array}{c}0.0289^{\{} \\
3\}\end{array}$ & $\begin{array}{c}0.316 \\
0\end{array}$ & $\begin{array}{c}0.0012^{\{1} \\
\}\end{array}$ & $\begin{array}{c}0.29 \\
86\end{array}$ & \multicolumn{2}{|c|}{$0.0023^{\{2\}}$} \\
\hline & 9.1230 & 0.3314 & 0.252 & $\begin{array}{c}0.0289^{\{} \\
3\}\end{array}$ & $\begin{array}{c}0.315 \\
7\end{array}$ & $0.0011^{\{1}$ & 0.29 & \multicolumn{2}{|c|}{$0.0023^{\{2\}}$} \\
\hline & 9.1065 & 0.3313 & 0.241 & $\begin{array}{c}0.0288^{\prime} \\
3\}\end{array}$ & $\begin{array}{c}0.315 \\
5\end{array}$ & $\underset{\}}{0.0011^{\{1}}$ & 0.29 & \multicolumn{2}{|c|}{$0.0023^{\{2\}}$} \\
\hline & 8.7947 & 0.3300 & 0.237 & $\begin{array}{c}0.0275^{\{} \\
3\}\end{array}$ & 0.312 & $\underset{\}}{0.0011^{\{1}}$ & 0.29 & \multicolumn{2}{|c|}{$0.0022^{\{2\}}$} \\
\hline & 8.2114 & 0.3283 & 0.231 & $\begin{array}{c}0.0269^{\{} \\
3\}\end{array}$ & $\begin{array}{c}0.310 \\
8\end{array}$ & $\begin{array}{c}0.0011^{\{1} \\
\end{array}$ & $\begin{array}{c}0.29 \\
58\end{array}$ & \multicolumn{2}{|c|}{$0.0021^{\{2\}}$} \\
\hline & 8.0695 & 0.3263 & $\begin{array}{c}0.229 \\
9\end{array}$ & $\begin{array}{c}0.0266^{\{} \\
3\}\end{array}$ & $\begin{array}{c}0.310 \\
0\end{array}$ & $0.0011^{\{1}$ & $\begin{array}{c}0.29 \\
33\end{array}$ & \multicolumn{2}{|c|}{$0.0020^{\{2\}}$} \\
\hline & $\sum R$ & & \multicolumn{2}{|c|}{$30^{\{3\}}$} & \multicolumn{2}{|c|}{$10^{\{1\}}$} & \multicolumn{3}{|c|}{$20^{\{2\}}$} \\
\hline & \multicolumn{2}{|c|}{$\begin{array}{c}\text { Estimated } \\
\text { parameters }\end{array}$} & $\hat{\lambda}$ & $\widehat{\alpha}$ & $\hat{\lambda}$ & $\widehat{\alpha}$ & $\hat{\lambda}$ & $\widehat{\boldsymbol{\theta}}$ & $\widehat{\alpha}$ \\
\hline & & & $\begin{array}{c}2.7 \\
0\end{array}$ & $\begin{array}{c}5.1 \\
1\end{array}$ & $\begin{array}{c}3.2 \\
5\end{array}$ & $\begin{array}{c}3.5 \\
0\end{array}$ & $\begin{array}{c}3.7 \\
5\end{array}$ & 3.9 & 3.29 \\
\hline II & 11 & al & Mle & MSE & MPS & MSE & $\begin{array}{l}\text { CV } \\
\text { MD }\end{array}$ & & SE \\
\hline & $\begin{array}{c}11.538 \\
6\end{array}$ & 0.3329 & $\begin{array}{c}0.351 \\
4\end{array}$ & $\begin{array}{c}0.0640^{\{} \\
3\}\end{array}$ & $\begin{array}{c}0.331 \\
2\end{array}$ & $\begin{array}{c}0.0001^{\{1} \\
.5\}\end{array}$ & $\begin{array}{c}0.34 \\
08\end{array}$ & & $01^{\{1.5}$ \\
\hline & $\begin{array}{c}10.924 \\
5\end{array}$ & 0.3328 & $\begin{array}{c}0.345 \\
?\end{array}$ & $\begin{array}{c}0.0550^{\{} \\
3\}\end{array}$ & 0.331 & $\begin{array}{c}0.00005 \\
\{1\}\end{array}$ & 0.34 & & $01^{\{2\}}$ \\
\hline & 9.0953 & 0.3326 & $\begin{array}{c}0.328 \\
7\end{array}$ & $\begin{array}{c}0.0343^{\prime} \\
3\}\end{array}$ & $\begin{array}{c}0.330 \\
7\end{array}$ & $\begin{array}{c}0.00005 \\
\{1\}\end{array}$ & $\begin{array}{c}0.34 \\
04\end{array}$ & & $01^{\{2\}}$ \\
\hline & 8.9342 & 0.3318 & $\begin{array}{c}0.303 \\
9\end{array}$ & $\begin{array}{c}0.0297^{\{} \\
3\}\end{array}$ & $\begin{array}{c}0.330 \\
7\end{array}$ & $\begin{array}{c}0.00004 \\
\{1\}\end{array}$ & 0.34 & 0.0 & $01^{\{2\}}$ \\
\hline 10 & 8.8230 & 0.3318 & $\begin{array}{c}0.300 \\
1\end{array}$ & $\begin{array}{c}0.0297^{\{} \\
3\}\end{array}$ & $\begin{array}{c}0.330 \\
7\end{array}$ & $\begin{array}{c}0.00004 \\
\{1\}\end{array}$ & 0.34 & 0.0 & $01^{\{2\}}$ \\
\hline $\mathbf{0}$ & 8.2663 & 0.3315 & $\begin{array}{c}0.291 \\
7\end{array}$ & $\begin{array}{c}0.0297^{\{} \\
3\}\end{array}$ & $\begin{array}{c}0.330 \\
6\end{array}$ & $\begin{array}{c}0.00004 \\
\{1\}\end{array}$ & $\begin{array}{c}0.33 \\
99\end{array}$ & & $01^{\{2\}}$ \\
\hline & 8.1689 & 0.3313 & $\begin{array}{c}0.273 \\
2\end{array}$ & $\begin{array}{c}0.0297^{\{} \\
3\}\end{array}$ & $\begin{array}{c}0.329 \\
0\end{array}$ & $\begin{array}{c}0.00002 \\
\{1\}\end{array}$ & $\begin{array}{c}0.33 \\
87\end{array}$ & 0.0 & $01^{\{2\}}$ \\
\hline & 7.9143 & 0.3313 & $\begin{array}{c}0.268 \\
1\end{array}$ & $\begin{array}{c}0.0297^{\{} \\
3\}\end{array}$ & $\begin{array}{c}0.329 \\
0\end{array}$ & $\begin{array}{c}0.00002 \\
\{1\}\end{array}$ & $\begin{array}{c}0.33 \\
87\end{array}$ & 0.0 & $01^{\{2\}}$ \\
\hline & 7.8467 & 0.3303 & $\begin{array}{c}0.258 \\
9\end{array}$ & $\begin{array}{c}0.0297^{\{} \\
3\}\end{array}$ & $\begin{array}{c}0.329 \\
0\end{array}$ & $\begin{array}{c}0.00002 \\
\{1\}\end{array}$ & $\begin{array}{c}0.33 \\
81\end{array}$ & 0.0 & $01^{\{2\}}$ \\
\hline & 7.2624 & 0.3296 & $\begin{array}{c}0.256 \\
0\end{array}$ & $\begin{array}{c}0.0296 \\
3\}\end{array}$ & $\begin{array}{c}0.328 \\
0\end{array}$ & $\begin{array}{c}0.00001 \\
\{1\}\end{array}$ & $\begin{array}{c}0.33 \\
76\end{array}$ & 0.0 & $01^{\{2\}}$ \\
\hline & ) $R$ & & & & & $5^{\{1\}}$ & & & \\
\hline
\end{tabular}

Table (3): Simulation results for $(\lambda=3.5, \theta=5, \alpha=2.5)$

\begin{tabular}{|c|c|c|c|c|c|c|c|c|c|c|c|}
\hline \multicolumn{3}{|c|}{$\begin{array}{c}\text { Estimated } \\
\text { parameters }\end{array}$} & $\hat{\lambda}$ & $\widehat{\boldsymbol{\theta}}$ & $\widehat{\boldsymbol{\alpha}}$ & $\hat{\lambda}$ & $\widehat{\boldsymbol{\theta}}$ & $\widehat{\boldsymbol{\alpha}}$ & $\hat{\lambda}$ & $\widehat{\boldsymbol{\theta}}$ & $\widehat{\boldsymbol{\alpha}}$ \\
\hline $\mathbf{N}$ & $\mathbf{T i}$ & Real & $\begin{array}{c}2.6 \\
9\end{array}$ & $\begin{array}{c}3.1 \\
1\end{array}$ & $\begin{array}{c}5.8 \\
1\end{array}$ & $\begin{array}{c}3.0 \\
7\end{array}$ & $\begin{array}{c}5.6 \\
9\end{array}$ & $\begin{array}{c}2.7 \\
1\end{array}$ & $\begin{array}{c}3.9 \\
6\end{array}$ & $\begin{array}{c}4.8 \\
0\end{array}$ & 2.34 \\
\hline
\end{tabular}




\begin{tabular}{|c|c|c|c|c|c|c|c|c|c|}
\hline & & & Mle & MSE & MPS & MSE & $\begin{array}{l}\text { CV } \\
\text { MD }\end{array}$ & & SE \\
\hline \multirow{10}{*}{35} & $\begin{array}{c}11.407 \\
7\end{array}$ & 0.2642 & 0.2755 & $\begin{array}{c}0.0473 \\
\{3\}\end{array}$ & $\begin{array}{c}0.264 \\
4\end{array}$ & $\begin{array}{c}0.00000 \\
1^{\{1\}}\end{array}$ & $\begin{array}{c}0.33 \\
07\end{array}$ & \multicolumn{2}{|c|}{$0.0064^{\{2\}}$} \\
\hline & $\begin{array}{c}11.348 \\
3\end{array}$ & 0.2629 & 0.2313 & $\begin{array}{c}0.0230 \\
\{3\}\end{array}$ & $\begin{array}{c}0.263 \\
2\end{array}$ & $\begin{array}{c}0.00000 \\
1^{\{1\}}\end{array}$ & $\begin{array}{c}0.32 \\
72\end{array}$ & \multicolumn{2}{|c|}{$0.0064^{\{2\}}$} \\
\hline & 9.9721 & 0.2627 & 0.2251 & $\begin{array}{c}0.0230 \\
\{3\}\end{array}$ & $\begin{array}{c}0.262 \\
8\end{array}$ & $\begin{array}{c}0.00000 \\
1^{\{1\}}\end{array}$ & $\begin{array}{c}0.32 \\
71\end{array}$ & \multicolumn{2}{|c|}{$0.0062^{\{2\}}$} \\
\hline & 9.6911 & 0.2589 & 0.2094 & $\begin{array}{c}0.0229 \\
\{3\}\end{array}$ & $\begin{array}{c}0.258 \\
9\end{array}$ & $\begin{array}{c}0.00000 \\
1^{\{1\}}\end{array}$ & $\begin{array}{c}0.31 \\
89\end{array}$ & \multicolumn{2}{|c|}{$0.0061^{\{2\}}$} \\
\hline & 9.1054 & 0.2559 & 0.1965 & $\begin{array}{c}0.0229 \\
\{3\}\end{array}$ & $\begin{array}{c}0.255 \\
8\end{array}$ & $\begin{array}{c}0.00000 \\
8^{\{1\}} \\
\end{array}$ & $\begin{array}{c}0.31 \\
61\end{array}$ & \multicolumn{2}{|c|}{$0.0053^{\{2\}}$} \\
\hline & 7.6634 & 0.2544 & 0.1833 & $\begin{array}{c}0.0229 \\
\{3\}\end{array}$ & $\begin{array}{c}0.254 \\
3\end{array}$ & $\begin{array}{c}0.00000 \\
7^{\{1\}}\end{array}$ & $\begin{array}{c}0.31 \\
46\end{array}$ & \multicolumn{2}{|c|}{$0.0046^{\{2\}}$} \\
\hline & 7.5934 & 0.2507 & 0.1691 & $\begin{array}{c}0.0229 \\
\{3\}\end{array}$ & $\begin{array}{c}0.250 \\
6\end{array}$ & $\begin{array}{c}0.00000 \\
6^{\{1\}}\end{array}$ & $\begin{array}{c}0.30 \\
27\end{array}$ & \multicolumn{2}{|c|}{$0.0042^{\{2\}}$} \\
\hline & 7.3583 & 0.2505 & 0.1667 & $\begin{array}{c}0.0228 \\
\{3\}\end{array}$ & $\begin{array}{c}0.250 \\
4\end{array}$ & $\begin{array}{c}0.00000 \\
5^{\{1\}}\end{array}$ & $\begin{array}{c}0.29 \\
51\end{array}$ & 0.0 & $40^{\{2\}}$ \\
\hline & 6.7842 & 0.2494 & 0.1578 & $\begin{array}{c}0.0225 \\
\{3\}\end{array}$ & $\begin{array}{c}0.249 \\
2\end{array}$ & $\begin{array}{c}0.00000 \\
4^{\{1\}}\end{array}$ & $\begin{array}{c}0.29 \\
46\end{array}$ & 0.0 & $27^{\{2\}}$ \\
\hline & 6.2422 & 0.2485 & 0.1355 & $\begin{array}{c}0.0224 \\
\{3\}\end{array}$ & $\begin{array}{c}0.249 \\
1\end{array}$ & $\begin{array}{c}0.00000 \\
1^{\{1\}}\end{array}$ & $\begin{array}{c}0.29 \\
15\end{array}$ & 0.0 & $27^{\{2\}}$ \\
\hline & $\sum R a$ & & & & & $0^{\{1\}}$ & & $0^{\{2\}}$ & \\
\hline & $\begin{array}{l}\text { Estima } \\
\text { parame }\end{array}$ & & $\hat{\lambda}$ & $\widehat{\alpha}$ & $\hat{\lambda}$ & $\widehat{\alpha}$ & $\hat{\lambda}$ & $\widehat{\boldsymbol{\theta}}$ & $\widehat{\alpha}$ \\
\hline & & & $\begin{array}{c}2.5 \\
9\end{array}$ & $\begin{array}{c}5.8 \\
2\end{array}$ & $\begin{array}{c}3.2 \\
1\end{array}$ & $\begin{array}{c}2.4 \\
8\end{array}$ & $\begin{array}{c}3.8 \\
5\end{array}$ & $\begin{array}{l}4.8 \\
2\end{array}$ & 2.37 \\
\hline $\mathbf{N}$ & Ti & Real & Mle & MSE & MPS & MSE & $\begin{array}{l}\text { CV } \\
\text { MD }\end{array}$ & & SE \\
\hline & $\begin{array}{c}12.525 \\
6\end{array}$ & 0.2643 & 0.2746 & $\begin{array}{c}0.0496 \\
\{3\}\end{array}$ & $\begin{array}{c}0.185 \\
4\end{array}$ & $\begin{array}{c}0.0062^{\{2} \\
\}\end{array}$ & $\begin{array}{c}0.32 \\
79\end{array}$ & 0.0 & $56^{\{1\}}$ \\
\hline & $\begin{array}{c}11.679 \\
9\end{array}$ & 0.2643 & 0.2559 & $\begin{array}{c}0.0302 \\
\{3\}\end{array}$ & $\begin{array}{c}0.185 \\
4\end{array}$ & $0.0062^{\{2}$ & $\begin{array}{c}0.32 \\
52\end{array}$ & 0.0 & $56^{\{1\}}$ \\
\hline & $\begin{array}{c}11.427 \\
7\end{array}$ & 0.2643 & 0.2491 & $\begin{array}{c}0.0239 \\
\{3\}\end{array}$ & $\begin{array}{c}0.185 \\
4\end{array}$ & $\begin{array}{c}0.0062^{\{2} \\
\}\end{array}$ & $\begin{array}{c}0.32 \\
46\end{array}$ & 0.0 & $56^{\{1\}}$ \\
\hline & 9.5865 & 0.2643 & 0.2290 & $\begin{array}{c}0.0226 \\
\{3\}\end{array}$ & $\begin{array}{c}0.185 \\
3\end{array}$ & $0.0062^{\{2}$ & $\begin{array}{c}70 \\
43\end{array}$ & 0.0 & $56^{\{1\}}$ \\
\hline & 9.4554 & 0.2641 & 0.2277 & $\begin{array}{c}0.0225 \\
\{3\}\end{array}$ & $\begin{array}{c}0.185 \\
3\end{array}$ & $\begin{array}{c}0.0062^{\{2} \\
\}\end{array}$ & $\begin{array}{c}0.32 \\
29\end{array}$ & 0.0 & $56^{\{1\}}$ \\
\hline 75 & 9.3709 & 0.2634 & 0.2273 & $\begin{array}{c}0.0223 \\
\{3\}\end{array}$ & $\begin{array}{c}0.184 \\
7\end{array}$ & $\begin{array}{c}0.0062^{\{2} \\
\}\end{array}$ & $\begin{array}{c}0.32 \\
21\end{array}$ & 0.0 & $54^{\{1\}}$ \\
\hline & 8.9241 & 0.2632 & 0.2254 & $\begin{array}{c}0.0223 \\
\{3\}\end{array}$ & $\begin{array}{c}0.184 \\
6\end{array}$ & $\begin{array}{c}0.0062^{\{2} \\
\}\end{array}$ & $\begin{array}{c}21 \\
0.32 \\
18\end{array}$ & 0.0 & $53^{\{1\}}$ \\
\hline & 8.7807 & 0.2630 & 0.2205 & $\begin{array}{c}0.0219 \\
\{3\}\end{array}$ & $\begin{array}{c}0.184 \\
5\end{array}$ & $\begin{array}{c}0.0062^{\{2} \\
\}\end{array}$ & $\begin{array}{c}0.32 \\
18\end{array}$ & 0.0 & $53^{\{1\}}$ \\
\hline & 8.6519 & 0.2620 & 0.2150 & $\begin{array}{c}0.0218 \\
\{3\}\end{array}$ & $\begin{array}{c}0.184 \\
2\end{array}$ & $\begin{array}{c}0.0061^{\{2} \\
\end{array}$ & $\begin{array}{c}0.32 \\
05\end{array}$ & 0.0 & $53^{\{1\}}$ \\
\hline & 8.6278 & 0.2606 & 0.2019 & $\begin{array}{c}0.0218 \\
\{3\}\end{array}$ & $\begin{array}{c}0.183 \\
9\end{array}$ & $\begin{array}{c}0.0060^{\{2} \\
\}\end{array}$ & $\begin{array}{c}0.31 \\
80\end{array}$ & 0.0 & $52^{\{1\}}$ \\
\hline & 1 & & & & & $\{2\}$ & & 0 & \\
\hline
\end{tabular}



Distribution.

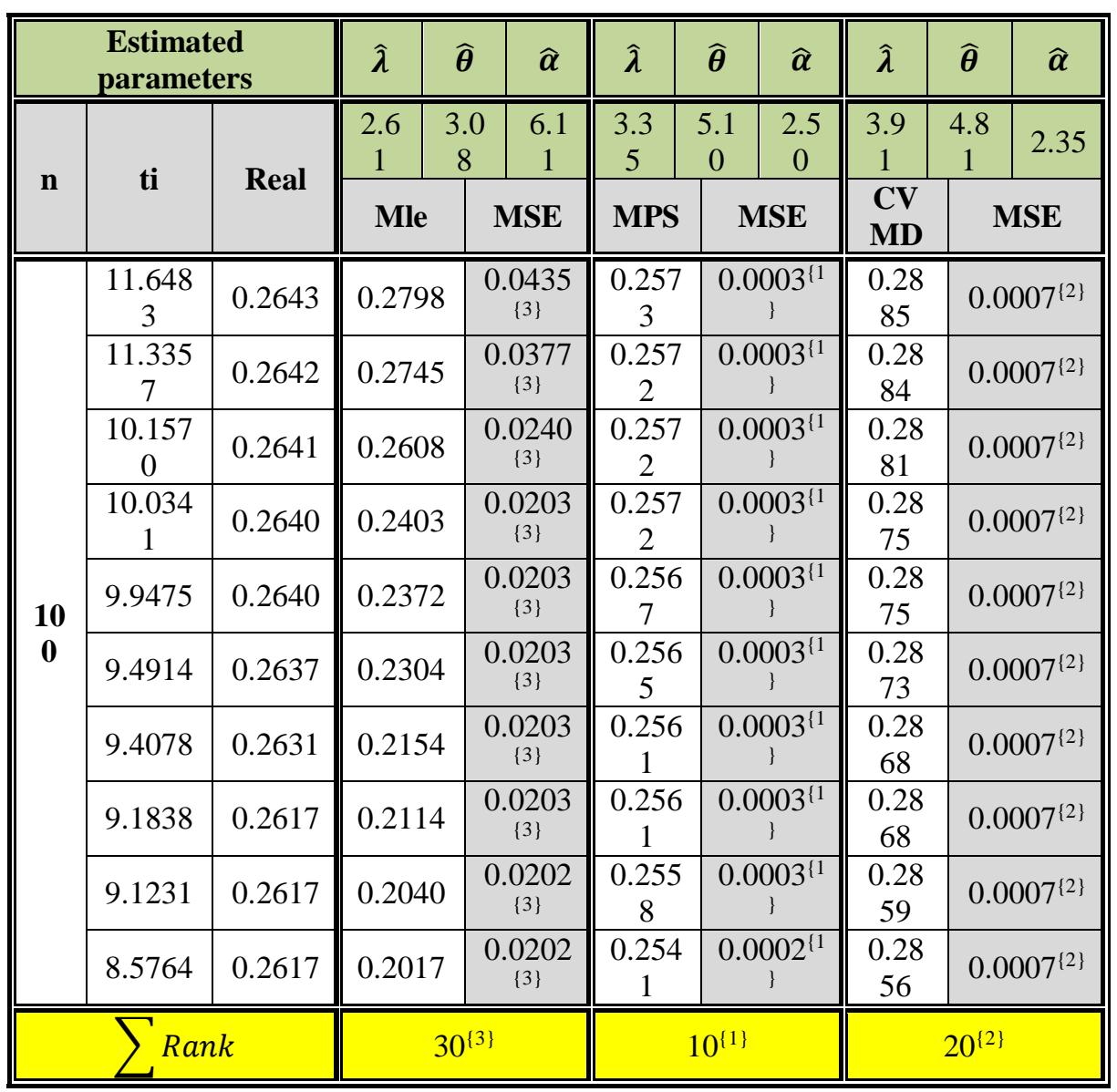

Table (4): Simulation results for $(\lambda=4, \theta=6.5, \alpha=3)$

\begin{tabular}{|c|c|c|c|c|c|c|c|c|c|c|c|}
\hline \multicolumn{3}{|c|}{$\begin{array}{l}\text { Estimated } \\
\text { parameters }\end{array}$} & $\hat{\lambda}$ & $\widehat{\boldsymbol{\theta}}$ & $\widehat{\boldsymbol{\alpha}}$ & $\hat{\lambda}$ & $\widehat{\boldsymbol{\theta}}$ & $\widehat{\alpha}$ & $\hat{\lambda}$ & $\widehat{\boldsymbol{\theta}}$ & $\widehat{\alpha}$ \\
\hline & \multirow[t]{2}{*}{ i } & \multirow[t]{2}{*}{ Real } & 2.64 & $\begin{array}{c}4.1 \\
2\end{array}$ & $\begin{array}{l}6 . \\
3 \\
2 \\
\end{array}$ & 7.04 & $\begin{array}{c}7.2 \\
5\end{array}$ & $\begin{array}{c}3.0 \\
6\end{array}$ & $\begin{array}{c}4.3 \\
1\end{array}$ & $\begin{array}{c}6.3 \\
6\end{array}$ & 2.84 \\
\hline & & & Mle & \multicolumn{2}{|c|}{ MSE } & MP & & $\begin{array}{l}\text { CV } \\
\text { MD }\end{array}$ & \multicolumn{2}{|c|}{ MSE } \\
\hline & 9 & 3 & $\begin{array}{c}0.197 \\
6\end{array}$ & \multicolumn{2}{|c|}{\begin{tabular}{|c|c|}
0.0206 \\
$3\}$
\end{tabular}} & $\begin{array}{l}.233 \\
2\end{array}$ & \multicolumn{2}{|c|}{$\begin{array}{c}0.000003^{1} \\
1\}\end{array}$} & $\begin{array}{l}0.27 \\
52\end{array}$ & \multicolumn{2}{|c|}{$0.0026^{\{2\}}$} \\
\hline & $\begin{array}{c}15.702 \\
3\end{array}$ & $\begin{array}{c}0.231 \\
6\end{array}$ & $\begin{array}{c}0.168 \\
2\end{array}$ & \multicolumn{2}{|c|}{$\begin{array}{c}0.0196^{\prime} \\
3\}\end{array}$} & $\begin{array}{c}0.232 \\
6\end{array}$ & \multicolumn{2}{|c|}{$\begin{array}{c}0.000003^{\prime} \\
1\}\end{array}$} & $\begin{array}{c}0.27 \\
30\end{array}$ & \multicolumn{2}{|c|}{$0.0026^{\{2\}}$} \\
\hline & $\begin{array}{c}13.034 \\
1\end{array}$ & $\begin{array}{c}0.230 \\
2\end{array}$ & $\begin{array}{c}0.164 \\
1\end{array}$ & \multicolumn{2}{|c|}{\begin{tabular}{|c|}
0.0196 \\
$3\}$
\end{tabular}} & $\begin{array}{c}0.230 \\
9\end{array}$ & \multicolumn{2}{|c|}{$\begin{array}{c}0.000003^{\{} \\
1\}\end{array}$} & $\begin{array}{c}0.27 \\
03\end{array}$ & \multicolumn{2}{|c|}{$0.0025^{\{2\}}$} \\
\hline & $\begin{array}{c}12.581 \\
6\end{array}$ & $\begin{array}{c}0.226 \\
4\end{array}$ & $\begin{array}{c}0.153 \\
8\end{array}$ & \multicolumn{2}{|c|}{$\begin{array}{c}0.0195^{\prime} \\
3\}\end{array}$} & $\begin{array}{c}0.226 \\
9\end{array}$ & \multicolumn{2}{|c|}{$\begin{array}{c}0.000003^{\{} \\
1\}\end{array}$} & $\begin{array}{c}0.26 \\
39\end{array}$ & \multicolumn{2}{|c|}{$0.0024^{\{2\}}$} \\
\hline 35 & $\begin{array}{c}11.699 \\
0\end{array}$ & $\begin{array}{c}0.223 \\
4\end{array}$ & $\begin{array}{c}0.145 \\
3\end{array}$ & \multicolumn{2}{|c|}{$\begin{array}{c}0.0193 \\
3\}\end{array}$} & $\begin{array}{c}0.223 \\
8\end{array}$ & \multicolumn{2}{|c|}{$\begin{array}{c}0.000003^{\prime} \\
1\}\end{array}$} & $\begin{array}{c}0.26 \\
31\end{array}$ & \multicolumn{2}{|c|}{$0.0023^{\{2\}}$} \\
\hline & 9.7573 & $\begin{array}{c}0.222 \\
0\end{array}$ & $\begin{array}{c}0.136 \\
7\end{array}$ & \multicolumn{2}{|c|}{$\begin{array}{c}0.0193^{\prime} \\
3\}\end{array}$} & $\begin{array}{c}0.222 \\
3\end{array}$ & \multicolumn{2}{|c|}{$\begin{array}{c}0.000002^{\{} \\
1\}\end{array}$} & $\begin{array}{c}0.26 \\
07\end{array}$ & \multicolumn{2}{|c|}{$0.0019^{\{2\}}$} \\
\hline & 9.6687 & $\begin{array}{c}0.220 \\
3\end{array}$ & $\begin{array}{c}0.127 \\
3\end{array}$ & \multicolumn{2}{|c|}{$\begin{array}{c}0.0192^{\prime} \\
3\}\end{array}$} & $\begin{array}{c}0.221 \\
7\end{array}$ & \multicolumn{2}{|c|}{$\begin{array}{c}0.000002^{\{} \\
1\}\end{array}$} & $\begin{array}{c}0.25 \\
49\end{array}$ & \multicolumn{2}{|c|}{$0.0017^{\{2\}}$} \\
\hline & 9.3736 & $\begin{array}{c}0.218 \\
5\end{array}$ & $\begin{array}{c}0.125 \\
7\end{array}$ & \multicolumn{2}{|c|}{$\begin{array}{c}0.0191^{\natural} \\
3\}\end{array}$} & $\begin{array}{c}0.218 \\
8\end{array}$ & \multicolumn{2}{|c|}{$\begin{array}{c}0.000002^{\{} \\
1\}\end{array}$} & $\begin{array}{c}0.24 \\
90\end{array}$ & \multicolumn{2}{|c|}{$0.0016^{\{2\}}$} \\
\hline
\end{tabular}




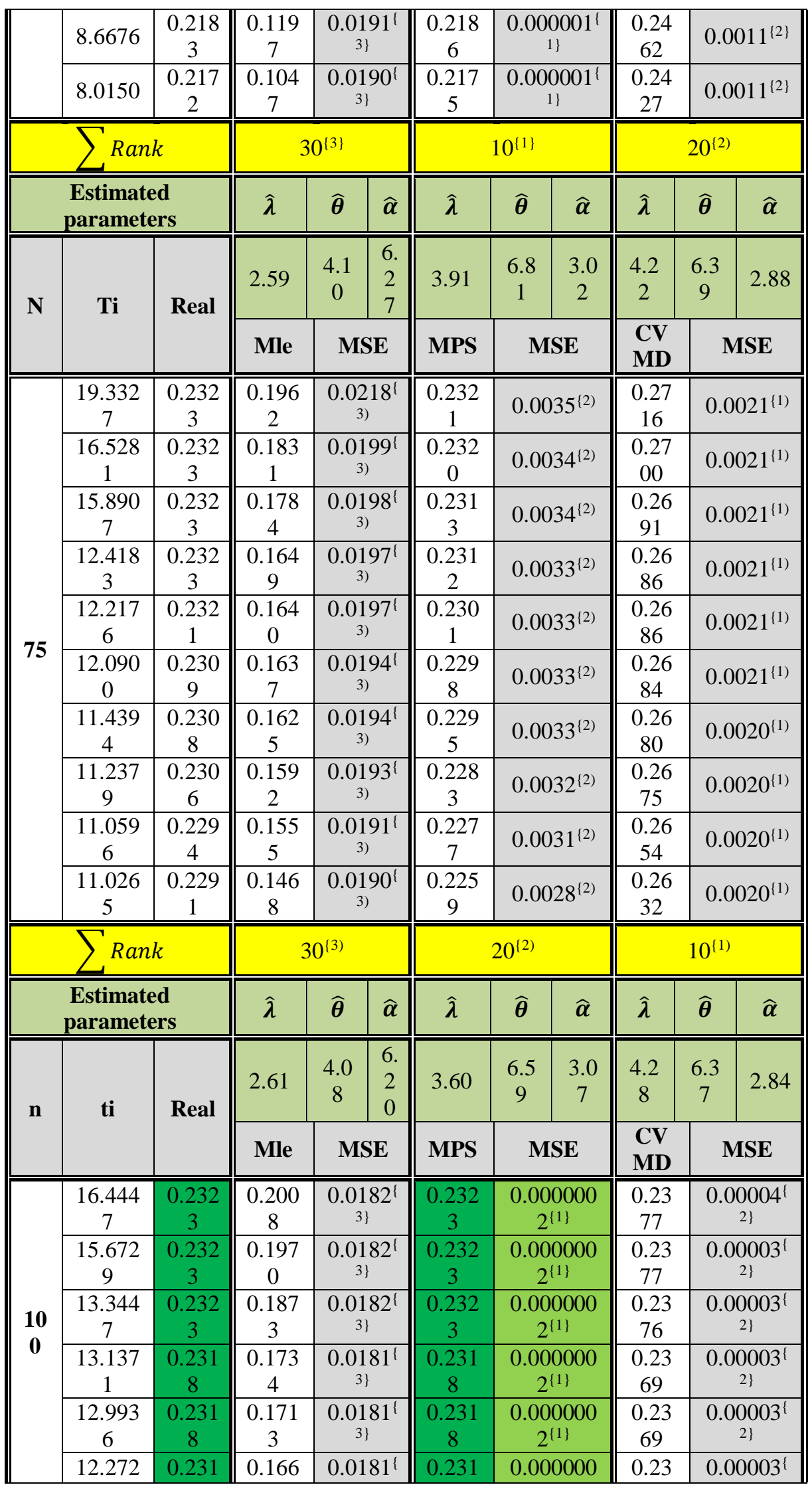


Statistical Properties \& Different Methods Of Estimation Of A New Extended Weighted Frechet Distribution.

\begin{tabular}{||c|c|c||c|c||c|c||c|c||}
\hline \multirow{4}{*}{2} & 3 & 7 & $3\}$ & 3 & $1^{\{1\}}$ & 65 & $2\}$ \\
\cline { 2 - 9 } & 12.145 & 0.230 & 0.156 & $0.0181^{\{}$ & 0.230 & 0.000000 & 0.23 & $0.00003^{\{}$ \\
5 & 7 & 6 & $3\}$ & 7 & $1^{\{1\}}$ & 65 & $2\}$ \\
\cline { 2 - 9 } & 11.813 & 0.230 & 0.153 & $0.0180^{\{}$ & 0.230 & 0.000000 & 0.23 & $0.00003^{\{}$ \\
1 & 7 & 9 & $3\}$ & 7 & $1^{\{1\}}$ & 63 & $2\}$ \\
\cline { 2 - 9 } & 11.724 & 0.230 & 0.149 & $0.0180^{\{}$ & 0.230 & 0.000000 & 0.23 & $0.00003^{\{}$ \\
& 6 & 0 & $3\}$ & 6 & $1^{\{1\}}$ & 63 & $2\}$ \\
\hline 10.956 & 0.230 & 0.147 & $0.0180^{\{}$ & 0.230 & 0.000000 & 0.23 & $0.00003^{\{}$ \\
2 & 5 & 5 & $3\}$ & 5 & $1^{\{1\}}$ & 55 & $2\}$ \\
\hline \hline
\end{tabular}

Table (5): Simulation results for $(\lambda=3, \theta=6.5, \alpha=4)$

\begin{tabular}{|c|c|c|c|c|c|c|c|c|c|c|c|}
\hline \multicolumn{3}{|c|}{$\begin{array}{c}\text { Estimated } \\
\text { parameters }\end{array}$} & $\hat{\lambda}$ & $\widehat{\boldsymbol{\theta}}$ & $\widehat{\alpha}$ & $\hat{\lambda}$ & $\widehat{\boldsymbol{\theta}}$ & $\widehat{\alpha}$ & $\hat{\lambda}$ & $\widehat{\boldsymbol{\theta}}$ & $\widehat{\alpha}$ \\
\hline \multirow[t]{2}{*}{ n } & & \multirow[b]{2}{*}{ ial } & \begin{tabular}{|c|}
2.3 \\
3 \\
\end{tabular} & $\begin{array}{c}4.0 \\
8\end{array}$ & $\begin{array}{c}6.6 \\
7\end{array}$ & $\begin{array}{c}2.7 \\
8\end{array}$ & $\begin{array}{c}7.1 \\
6 \\
\end{array}$ & $\begin{array}{c}3.1 \\
9\end{array}$ & $\begin{array}{c}3.2 \\
5\end{array}$ & $\begin{array}{c}6.3 \\
0\end{array}$ & 3.74 \\
\hline & & & Mle & \multicolumn{2}{|c|}{ MSE } & MPS & \multicolumn{2}{|c|}{ MSE } & $\begin{array}{l}\text { CV } \\
\text { MD }\end{array}$ & \multicolumn{2}{|c|}{ MSE } \\
\hline \multirow{10}{*}{35} & $\begin{array}{c}20.566 \\
5\end{array}$ & 0.1776 & $\begin{array}{c}0.203 \\
5\end{array}$ & \multicolumn{2}{|c|}{$\begin{array}{c}0.0251^{1} \\
3\}\end{array}$} & $\begin{array}{c}0.110 \\
0\end{array}$ & \multicolumn{2}{|c|}{$\begin{array}{c}0.0051^{12} \\
\}\end{array}$} & $\begin{array}{c}0.21 \\
23\end{array}$ & \multicolumn{2}{|c|}{$0.0018^{\{1\}}$} \\
\hline & $\begin{array}{c}20.348 \\
0\end{array}$ & 0.1773 & $\begin{array}{c}0.174 \\
3\end{array}$ & \multicolumn{2}{|c|}{$\begin{array}{c}0.0078^{\prime} \\
3\}\end{array}$} & $\begin{array}{c}0.109 \\
9\end{array}$ & \multicolumn{2}{|c|}{$\begin{array}{c}0.0050^{\{2} \\
\}\end{array}$} & $\begin{array}{c}0.21 \\
22\end{array}$ & \multicolumn{2}{|c|}{$0.0018^{\{1}$} \\
\hline & $\begin{array}{c}16.159 \\
4\end{array}$ & 0.1740 & $\begin{array}{c}0.169 \\
9\end{array}$ & & $\begin{array}{l}0078^{\prime} \\
3\}\end{array}$ & $\begin{array}{c}0.109 \\
6\end{array}$ & & $\begin{array}{l}050^{12} \\
1\end{array}$ & $\begin{array}{c}0.20 \\
95 \\
\end{array}$ & & $018\{1\}$ \\
\hline & $\begin{array}{c}15.449 \\
5\end{array}$ & 0.1716 & $\begin{array}{c}0.158 \\
6\end{array}$ & & $\begin{array}{l}0078^{1} \\
3\}\end{array}$ & $\begin{array}{c}0.109 \\
6\end{array}$ & & $048^{\{2}$ & $\begin{array}{c}0.20 \\
79\end{array}$ & & $017^{\{1\}}$ \\
\hline & $\begin{array}{c}14.073 \\
4\end{array}$ & 0.1699 & $\begin{array}{c}0.14 \mathrm{C} \\
1\end{array}$ & & $\begin{array}{l}0078^{\prime} \\
3\}\end{array}$ & $\begin{array}{c}0.108 \\
5\end{array}$ & & $046^{12}$ & $\begin{array}{c}0.20 \\
63\end{array}$ & & $016^{\{1\}}$ \\
\hline & $\begin{array}{c}11.111 \\
6\end{array}$ & 0.1671 & $\begin{array}{c}0.139 \\
3\end{array}$ & & $\begin{array}{l}0078^{1} \\
3\}\end{array}$ & $\begin{array}{c}0.107 \\
1\end{array}$ & & $045^{\{2}$ & $\begin{array}{c}0.20 \\
03\end{array}$ & & $15^{\{1\}}$ \\
\hline & $\begin{array}{c}10.979 \\
4\end{array}$ & 0.1664 & $\begin{array}{c}0.128 \\
5\end{array}$ & & $\begin{array}{l}0078^{1} \\
3\}\end{array}$ & $\begin{array}{c}0.106 \\
8\end{array}$ & & $043^{12}$ & $\begin{array}{c}0.19 \\
40\end{array}$ & & $012^{\{1\}}$ \\
\hline & $\begin{array}{c}10.541 \\
3\end{array}$ & 0.1657 & $\begin{array}{c}0.126 \\
7\end{array}$ & & $\begin{array}{l}0078^{1} \\
3\}\end{array}$ & $\begin{array}{c}0.106 \\
0\end{array}$ & & $\overline{043^{12}}$ & $\begin{array}{c}0.19 \\
20\end{array}$ & & $011^{\{1\}}$ \\
\hline & 9.5079 & 0.1648 & $\begin{array}{c}0.119 \\
8\end{array}$ & & $\begin{array}{l}0078^{\{} \\
3\}\end{array}$ & $\begin{array}{c}0.103 \\
8\end{array}$ & & $042^{\{2}$ & $\begin{array}{c}0.18 \\
68\end{array}$ & & $9^{\{1\}}$ \\
\hline & 8.5734 & 0.1626 & $\begin{array}{c}0.102 \\
6 \\
\end{array}$ & & $\begin{array}{l}0076^{1} \\
3\}\end{array}$ & $\begin{array}{c}0.103 \\
1 \\
\end{array}$ & & $\begin{array}{l}041^{\{2} \\
\}\end{array}$ & $\begin{array}{c}0.18 \\
44 \\
\end{array}$ & & $07^{\{1\}}$ \\
\hline \multicolumn{3}{|c|}{$\sum$ Rank } & \multicolumn{3}{|c|}{$30^{\{3\}}$} & \multicolumn{3}{|c|}{$20^{\{2\}}$} & \multicolumn{3}{|c|}{$10^{\{1}$} \\
\hline \multicolumn{3}{|c|}{$\begin{array}{c}\text { Estimated } \\
\text { parameters }\end{array}$} & $\hat{\lambda}$ & $\widehat{\boldsymbol{\theta}}$ & $\widehat{\alpha}$ & $\hat{\lambda}$ & $\widehat{\boldsymbol{\theta}}$ & $\widehat{\alpha}$ & $\hat{\lambda}$ & $\widehat{\boldsymbol{\theta}}$ & $\widehat{\alpha}$ \\
\hline \multirow[b]{2}{*}{ n } & \multirow[b]{2}{*}{ Ti } & \multirow[b]{2}{*}{ Re } & $\begin{array}{c}2.3 \\
0\end{array}$ & $\begin{array}{c}4.0 \\
6\end{array}$ & $\begin{array}{c}6.5 \\
6\end{array}$ & $\begin{array}{c}2.6 \\
9\end{array}$ & $\begin{array}{c}6.8 \\
1\end{array}$ & $\begin{array}{c}3.2 \\
0\end{array}$ & $\begin{array}{c}3.1 \\
8\end{array}$ & $\begin{array}{c}6.3 \\
4\end{array}$ & 3.78 \\
\hline & & & Mle & & ISE & MPS & & ISE & $\begin{array}{l}\text { CV } \\
\text { MD } \\
\end{array}$ & & ISE \\
\hline \multirow{3}{*}{75} & $\begin{array}{c}25.788 \\
4\end{array}$ & 0 & $\begin{array}{c}0.204 \\
6\end{array}$ & & $\begin{array}{l}0269^{\prime} \\
3\}\end{array}$ & $\begin{array}{c}0.170 \\
5\end{array}$ & & $001^{\{1}$ & $\begin{array}{c}0.21 \\
09\end{array}$ & \multicolumn{2}{|c|}{$0.0014^{\{2}$} \\
\hline & $\begin{array}{c}21.625 \\
7\end{array}$ & 0.1772 & $\begin{array}{c}0.192 \\
5\end{array}$ & & $\begin{array}{l}0168^{\prime} \\
3\}\end{array}$ & $\begin{array}{c}0.170 \\
3\end{array}$ & & 0005 & $\begin{array}{c}0.21 \\
02\end{array}$ & \multicolumn{2}{|c|}{$0.0014^{\{2\}}$} \\
\hline & $\begin{array}{c}20.640 \\
9\end{array}$ & 0.1770 & $\begin{array}{c}0.187 \\
9\end{array}$ & & $\begin{array}{l}0135^{\prime} \\
3\}\end{array}$ & $\begin{array}{c}0.170 \\
1\end{array}$ & & 0005 & $\begin{array}{c}0.20 \\
99\end{array}$ & & $014^{\{2\}}$ \\
\hline
\end{tabular}


Mahdi Wahhab Neamah ${ }^{1}$, Nahla Hadi Abdul-Sahib ${ }^{2}$

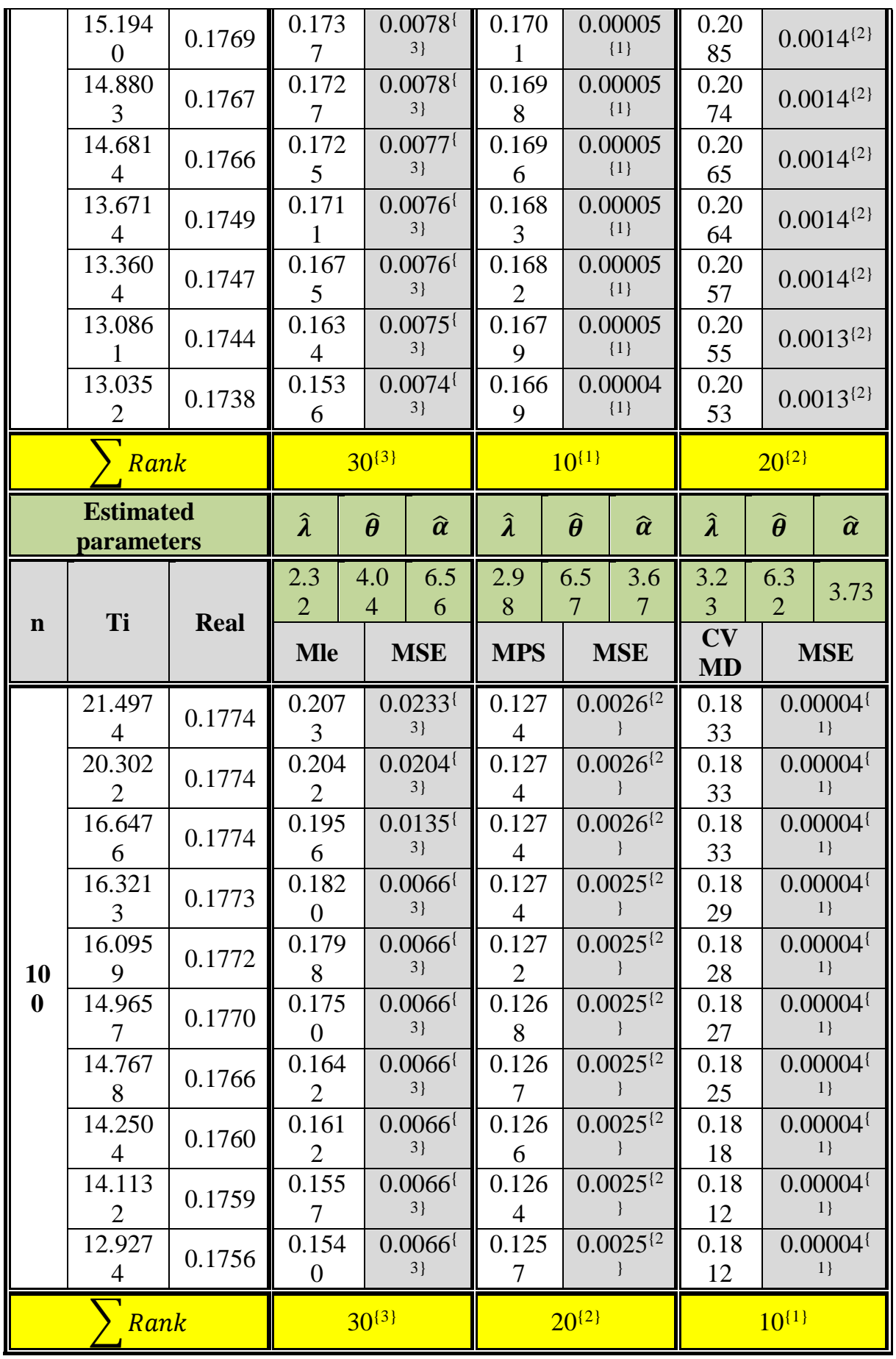

Table (6): Simulation results for $(\lambda=2, \theta=4, \alpha=2)$

\begin{tabular}{|c|c|c|c|c|c|c|c|c|c|c|c|}
\hline \multicolumn{3}{|c|}{$\begin{array}{c}\text { Estimated } \\
\text { parameters }\end{array}$} & $\hat{\lambda}$ & $\widehat{\boldsymbol{\theta}}$ & $\widehat{\alpha}$ & $\hat{\lambda}$ & $\widehat{\boldsymbol{\theta}}$ & $\widehat{\alpha}$ & $\hat{\lambda}$ & $\widehat{\boldsymbol{\theta}}$ & $\widehat{\alpha}$ \\
\hline \multirow{2}{*}{$\mathbf{N}$} & \multirow{2}{*}{$\mathbf{T i}$} & \multirow{2}{*}{ Real } & $\begin{array}{c}2.3 \\
9 \\
\end{array}$ & $\begin{array}{c}2.1 \\
9 \\
\end{array}$ & $\begin{array}{c}5.4 \\
7 \\
\end{array}$ & $\begin{array}{c}1.8 \\
9 \\
\end{array}$ & $\begin{array}{c}3.5 \\
7 \\
\end{array}$ & $\begin{array}{c}1.6 \\
4 \\
\end{array}$ & $\begin{array}{c}2.6 \\
5 \\
\end{array}$ & $\begin{array}{c}3.4 \\
0\end{array}$ & 1.92 \\
\hline & & & Mle & \multicolumn{2}{|c|}{ MSE } & MPS & \multicolumn{2}{|c|}{ MSE } & $\begin{array}{l}\text { CV } \\
\text { MD }\end{array}$ & \multicolumn{2}{|c|}{ MSE } \\
\hline$\overline{35}$ & 8.1785 & 0.1833 & 0.409 & & $\overline{486^{1}}$ & $\overline{0.179}$ & & $\overline{0005}$ & 0.33 & \multicolumn{2}{|c|}{$0.0346^{12}$} \\
\hline
\end{tabular}


Statistical Properties \& Different Methods Of Estimation Of A New Extended Weighted Frechet Distribution.

\begin{tabular}{|c|c|c|c|c|c|c|c|c|c|c|c|}
\hline & & & 3 & & 3\} & 8 & & \{1\} & 71 & & \\
\hline & 8.1609 & 0.1832 & $\begin{array}{c}0.355 \\
4\end{array}$ & & $\begin{array}{l}0831^{\prime} \\
3\}\end{array}$ & $\begin{array}{c}0.179 \\
8\end{array}$ & & 0005 & $\begin{array}{c}0.33 \\
23\end{array}$ & & $344^{\{2\}}$ \\
\hline & 7.6595 & 0.1830 & $\begin{array}{c}0.345 \\
3\end{array}$ & & $\begin{array}{l}0732^{\prime} \\
3\}\end{array}$ & $\begin{array}{c}0.179 \\
6\end{array}$ & & 0005 & $\begin{array}{c}0.33 \\
02\end{array}$ & & $336^{\{2\}}$ \\
\hline & 7.5309 & 0.1824 & $\begin{array}{c}0.318 \\
2 \\
\end{array}$ & & $\begin{array}{l}0500^{\{} \\
3\}\end{array}$ & $\begin{array}{c}0.179 \\
4 \\
\end{array}$ & & $\begin{array}{l}0005 \\
\{1\}\end{array}$ & $\begin{array}{c}0.32 \\
82 \\
\end{array}$ & & $327^{\{2\}}$ \\
\hline & 7.2291 & 0.1823 & $\begin{array}{c}0.294 \\
7 \\
\end{array}$ & & $\begin{array}{l}0338^{\prime} \\
3\} \\
\end{array}$ & $\begin{array}{c}0.179 \\
3\end{array}$ & & 0004 & $\begin{array}{c}0.32 \\
05\end{array}$ & & $297^{\{2\}}$ \\
\hline & 6.2637 & 0.1822 & $\begin{array}{c}0.270 \\
4\end{array}$ & & $\begin{array}{l}0204^{\prime} \\
2\}\end{array}$ & $\begin{array}{c}0.179 \\
1\end{array}$ & & $\begin{array}{l}0004 \\
\{1\}\end{array}$ & $\begin{array}{c}0.31 \\
08\end{array}$ & & $239^{\{3\}}$ \\
\hline & 6.2082 & 0.1819 & $\begin{array}{c}0.243 \\
7\end{array}$ & & $\begin{array}{l}0099^{\prime} \\
2\}\end{array}$ & $\begin{array}{c}0.178 \\
7\end{array}$ & & 0003 & $\begin{array}{c}0.30 \\
01\end{array}$ & & $236^{\{3\}}$ \\
\hline & 6.0160 & 0.1814 & $\begin{array}{c}0.239 \\
3\end{array}$ & & $\begin{array}{l}0085^{1} \\
2\}\end{array}$ & $\begin{array}{c}0.178 \\
7\end{array}$ & & 0003 & $\begin{array}{c}0.28 \\
35\end{array}$ & & $203^{\{3\}}$ \\
\hline & 5.5093 & 0.1813 & $\begin{array}{c}0.222 \\
5\end{array}$ & & $\begin{array}{l}0076^{1} \\
2\}\end{array}$ & $\begin{array}{c}0.178 \\
1\end{array}$ & & 0003 & $\begin{array}{c}0.28 \\
34\end{array}$ & & $158^{\{3\}}$ \\
\hline & 4.9867 & 0.1796 & $\begin{array}{c}0.181 \\
9\end{array}$ & & $\begin{array}{l}0074^{4} \\
2\}\end{array}$ & $\begin{array}{c}0.177 \\
3\end{array}$ & & 0003 & $\begin{array}{c}0.27 \\
37\end{array}$ & & $139^{\{3\}}$ \\
\hline & $\sum R a$ & & & 2.5 & & & $0^{\{1\}}$ & & & $5^{\{2.5}$ & \\
\hline & $\begin{array}{c}\text { Estima } \\
\text { parame }\end{array}$ & & $\hat{\lambda}$ & $\widehat{\boldsymbol{\theta}}$ & $\widehat{\alpha}$ & $\hat{\lambda}$ & $\widehat{\boldsymbol{\theta}}$ & $\widehat{\alpha}$ & $\hat{\lambda}$ & $\widehat{\boldsymbol{\theta}}$ & $\widehat{\alpha}$ \\
\hline N & $\mathbf{T}$ & & $\begin{array}{c}2.1 \\
5\end{array}$ & $\begin{array}{l}2.2 \\
0\end{array}$ & $\begin{array}{c}5.9 \\
2 \\
\end{array}$ & $\begin{array}{c}1.9 \\
3 \\
\end{array}$ & $\begin{array}{l}3.8 \\
4\end{array}$ & $\begin{array}{c}1.9 \\
2\end{array}$ & $\begin{array}{c}2.6 \\
4 \\
\end{array}$ & $\begin{array}{c}3.3 \\
4 \\
\end{array}$ & 1.94 \\
\hline N & 11 & Neal & Mle & & MSE & MPS & & ISE & $\begin{array}{l}\text { CV } \\
\text { MD }\end{array}$ & & ISE \\
\hline & 8.4581 & 0.1831 & $\begin{array}{c}0.385 \\
0\end{array}$ & & $\begin{array}{l}1328^{\prime} \\
3\}\end{array}$ & $\begin{array}{c}0.148 \\
8\end{array}$ & & $023^{\{1}$ & $\begin{array}{c}0.33 \\
44\end{array}$ & & $332^{\{2\}}$ \\
\hline & 8.2550 & 0.1830 & $\begin{array}{c}0.369 \\
4\end{array}$ & & $\begin{array}{l}1107^{\prime} \\
3\}\end{array}$ & $\begin{array}{c}0.148 \\
7\end{array}$ & & $021^{\{1}$ & $\begin{array}{c}0.33 \\
44\end{array}$ & & $331^{\{2\}}$ \\
\hline & 8.1843 & 0.1827 & $\begin{array}{c}0.361 \\
6\end{array}$ & & $\begin{array}{l}1011^{\ell} \\
3\}\end{array}$ & $\begin{array}{c}0.148 \\
7\end{array}$ & & $020^{\{1}$ & $\begin{array}{c}0.33 \\
38\end{array}$ & & $331^{\{2\}}$ \\
\hline & 7.4805 & 0.1824 & $\begin{array}{c}0.334 \\
6 \\
\end{array}$ & & $\begin{array}{l}0718^{\{} \\
3\}\end{array}$ & $\begin{array}{c}0.148 \\
7\end{array}$ & & $018^{\{1}$ & $\begin{array}{c}0.33 \\
19 \\
\end{array}$ & & $330^{\{2\}}$ \\
\hline & 7.4152 & 0.1822 & $\begin{array}{c}0.332 \\
6\end{array}$ & & $\begin{array}{l}0699\{ \\
3\}\end{array}$ & $\begin{array}{c}0.148 \\
5\end{array}$ & & $017^{\{1}$ & $\begin{array}{c}0.33 \\
14\end{array}$ & & $328^{\{2\}}$ \\
\hline 75 & 7.3718 & 0.1820 & $\begin{array}{c}0.332 \\
0\end{array}$ & & $\begin{array}{l}0693^{\prime} \\
3\}\end{array}$ & $\begin{array}{c}0.148 \\
2\end{array}$ & & $017^{\{1}$ & $\begin{array}{c}0.33 \\
06\end{array}$ & & $328^{\{2\}}$ \\
\hline & 7.1257 & 0.1818 & $\begin{array}{c}0.329 \\
2\end{array}$ & & $\begin{array}{l}0666^{1} \\
3\}\end{array}$ & $\begin{array}{c}0.147 \\
8\end{array}$ & & $017^{\{1}$ & $\begin{array}{c}0.33 \\
04\end{array}$ & & $327^{\{2\}}$ \\
\hline & 7.0404 & 0.1807 & $\begin{array}{c}0.321 \\
7\end{array}$ & & $\begin{array}{l}0596^{1} \\
3\}\end{array}$ & $\begin{array}{c}0.147 \\
8\end{array}$ & & $017^{\{1}$ & $\begin{array}{c}0.33 \\
03\end{array}$ & 0.0 & $317^{\{2\}}$ \\
\hline & 6.9611 & 0.1803 & $\begin{array}{c}0.313 \\
0\end{array}$ & & $\begin{array}{l}0522^{\prime} \\
3\}\end{array}$ & $\begin{array}{c}0.147 \\
7\end{array}$ & & $017^{\{1}$ & $\begin{array}{c}0.32 \\
99\end{array}$ & & $311^{\{2\}}$ \\
\hline & 6.9460 & 0.1798 & $\begin{array}{c}0.291 \\
6 \\
\end{array}$ & & $\begin{array}{l}0359^{\prime} \\
3\}\end{array}$ & $\begin{array}{c}0.147 \\
1 \\
\end{array}$ & & $\begin{array}{l}017^{11} \\
\}\end{array}$ & $\begin{array}{c}0.31 \\
91 \\
\end{array}$ & 0.0 & $305^{\{2\}}$ \\
\hline & $\sum R$ & & & $0^{\{3\}}$ & & & $0^{\{1\}}$ & & & $20^{\{2\}}$ & \\
\hline & $\begin{array}{l}\text { Estima } \\
\text { parame }\end{array}$ & & $\hat{\lambda}$ & $\widehat{\boldsymbol{\theta}}$ & $\widehat{\boldsymbol{\alpha}}$ & $\hat{\lambda}$ & $\widehat{\boldsymbol{\theta}}$ & $\widehat{\boldsymbol{\alpha}}$ & $\hat{\lambda}$ & $\widehat{\boldsymbol{\theta}}$ & $\widehat{\alpha}$ \\
\hline n & Ti & Real & $\begin{array}{c}2.1 \\
6\end{array}$ & $\begin{array}{c}2.1 \\
9 \\
\end{array}$ & $\begin{array}{c}5.7 \\
6\end{array}$ & $\begin{array}{c}1.9 \\
1\end{array}$ & $\begin{array}{c}3.8 \\
5 \\
\end{array}$ & $\begin{array}{c}1.8 \\
8\end{array}$ & $\begin{array}{c}2.6 \\
6\end{array}$ & $\begin{array}{c}3.3 \\
4\end{array}$ & 1.93 \\
\hline
\end{tabular}




\begin{tabular}{|c|c|c|c|c|c|c|c|c|}
\hline & & & Mle & MSE & MPS & MSE & $\begin{array}{l}\text { CV } \\
\text { MD } \\
\end{array}$ & MSE \\
\hline \multirow{10}{*}{$\begin{array}{c}10 \\
0\end{array}$} & 8.2464 & 0.1833 & $\begin{array}{c}0.389 \\
2\end{array}$ & $\begin{array}{c}0.1286^{1} \\
3\}\end{array}$ & $\begin{array}{c}0.149 \\
2\end{array}$ & $\begin{array}{c}0.0022^{\{1} \\
\}\end{array}$ & $\begin{array}{c}0.25 \\
41 \\
\end{array}$ & $0.0066^{\{2\}}$ \\
\hline & 8.1572 & 0.1832 & $\begin{array}{c}0.387 \\
0\end{array}$ & $\underset{3\}}{0.1235^{\prime}}$ & $\begin{array}{c}0.149 \\
1\end{array}$ & $0.0020^{\{1}$ & $\begin{array}{c}0.25 \\
41\end{array}$ & $0.0066^{\{2\}}$ \\
\hline & 7.7388 & 0.1831 & $\begin{array}{c}0.376 \\
9\end{array}$ & $\begin{array}{c}0.1067 \\
3\}\end{array}$ & $\begin{array}{c}0.149 \\
1\end{array}$ & $\begin{array}{c}0.0019^{\{1} \\
\}\end{array}$ & $\begin{array}{c}0.25 \\
41\end{array}$ & $0.0065^{\{2\}}$ \\
\hline & 7.6866 & 0.1830 & $\begin{array}{c}0.353 \\
4\end{array}$ & $\begin{array}{c}0.0779^{\prime} \\
3\}\end{array}$ & $\begin{array}{c}0.149 \\
1\end{array}$ & $0.0017^{\{1}$ & $\begin{array}{c}0.25 \\
34\end{array}$ & $0.0064^{\{2\}}$ \\
\hline & 7.6486 & 0.1830 & $\begin{array}{c}0.349 \\
2\end{array}$ & $\begin{array}{c}0.0734 \\
3\}\end{array}$ & $\begin{array}{c}0.149 \\
1\end{array}$ & $0.0016^{\{1}$ & $\begin{array}{c}0.25 \\
34\end{array}$ & $0.0063^{\{2\}}$ \\
\hline & 7.4333 & 0.1829 & $\begin{array}{c}0.339 \\
4\end{array}$ & $\begin{array}{c}0.0639^{\prime} \\
3\}\end{array}$ & $\begin{array}{c}0.149 \\
0\end{array}$ & $\begin{array}{c}0.0016^{\{1} \\
\}\end{array}$ & $\begin{array}{c}0.25 \\
28\end{array}$ & $0.0063^{\{2\}}$ \\
\hline & 7.3909 & 0.1829 & $\begin{array}{c}0.316 \\
4\end{array}$ & $\begin{array}{c}0.0444 \\
3\}\end{array}$ & $\begin{array}{c}0.148 \\
7\end{array}$ & $\begin{array}{c}0.0016^{\{1} \\
\}\end{array}$ & $\begin{array}{c}0.25 \\
19\end{array}$ & $0.0063^{\{2\}}$ \\
\hline & 7.2723 & 0.1829 & $\begin{array}{c}0.309 \\
8\end{array}$ & $\begin{array}{c}0.0396^{1} \\
3\}\end{array}$ & $\begin{array}{c}0.148 \\
6\end{array}$ & $\begin{array}{c}0.0016^{\{1} \\
\end{array}$ & $\begin{array}{c}0.25 \\
19\end{array}$ & $0.0062^{\{2\}}$ \\
\hline & 7.2389 & 0.1824 & $\begin{array}{c}0.297 \\
5\end{array}$ & $\underset{3\}}{0.0315^{1}}$ & $\begin{array}{c}0.148 \\
6\end{array}$ & $0.0016^{\{1}$ & $\begin{array}{c}0.25 \\
19\end{array}$ & $0.0062^{\{2\}}$ \\
\hline & 6.9134 & 0.1822 & $\begin{array}{c}0.293 \\
6 \\
\end{array}$ & $\begin{array}{c}0.0292^{\prime} \\
3\}\end{array}$ & $\begin{array}{c}0.148 \\
3 \\
\end{array}$ & $\begin{array}{c}0.0016^{11} \\
\quad\end{array}$ & $\begin{array}{c}0.25 \\
16 \\
\end{array}$ & $0.0060^{\{2\}}$ \\
\hline \multicolumn{3}{|c|}{$\sum$ Rank } & \multicolumn{2}{|c|}{$30^{\{3\}}$} & \multicolumn{2}{|c|}{$10^{\{1\}}$} & \multicolumn{2}{|c|}{$20^{\{2\}}$} \\
\hline
\end{tabular}

Table (7): Simulation results for $(\lambda=4.5, \theta=5, \alpha=3)$

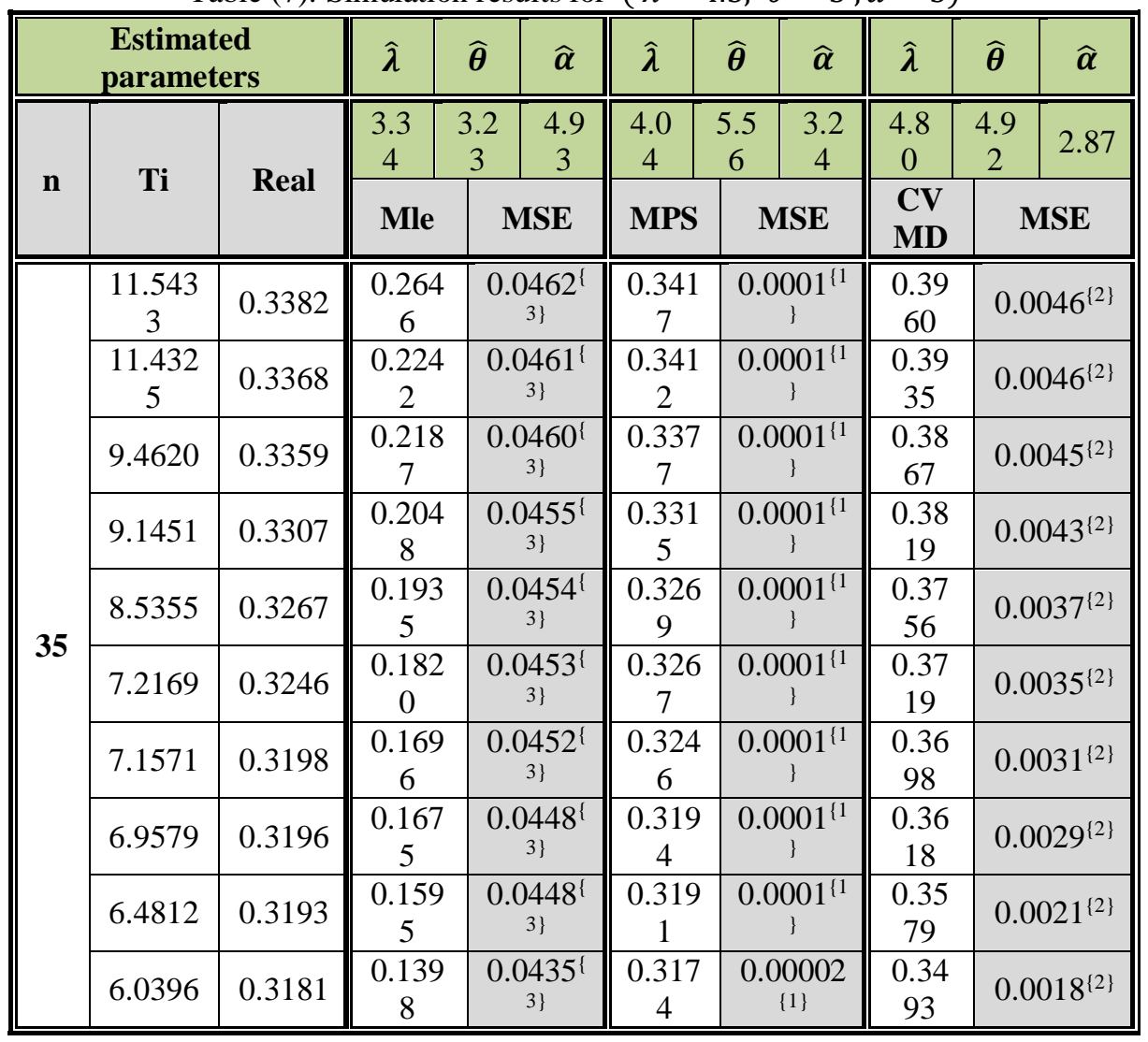



Distribution.

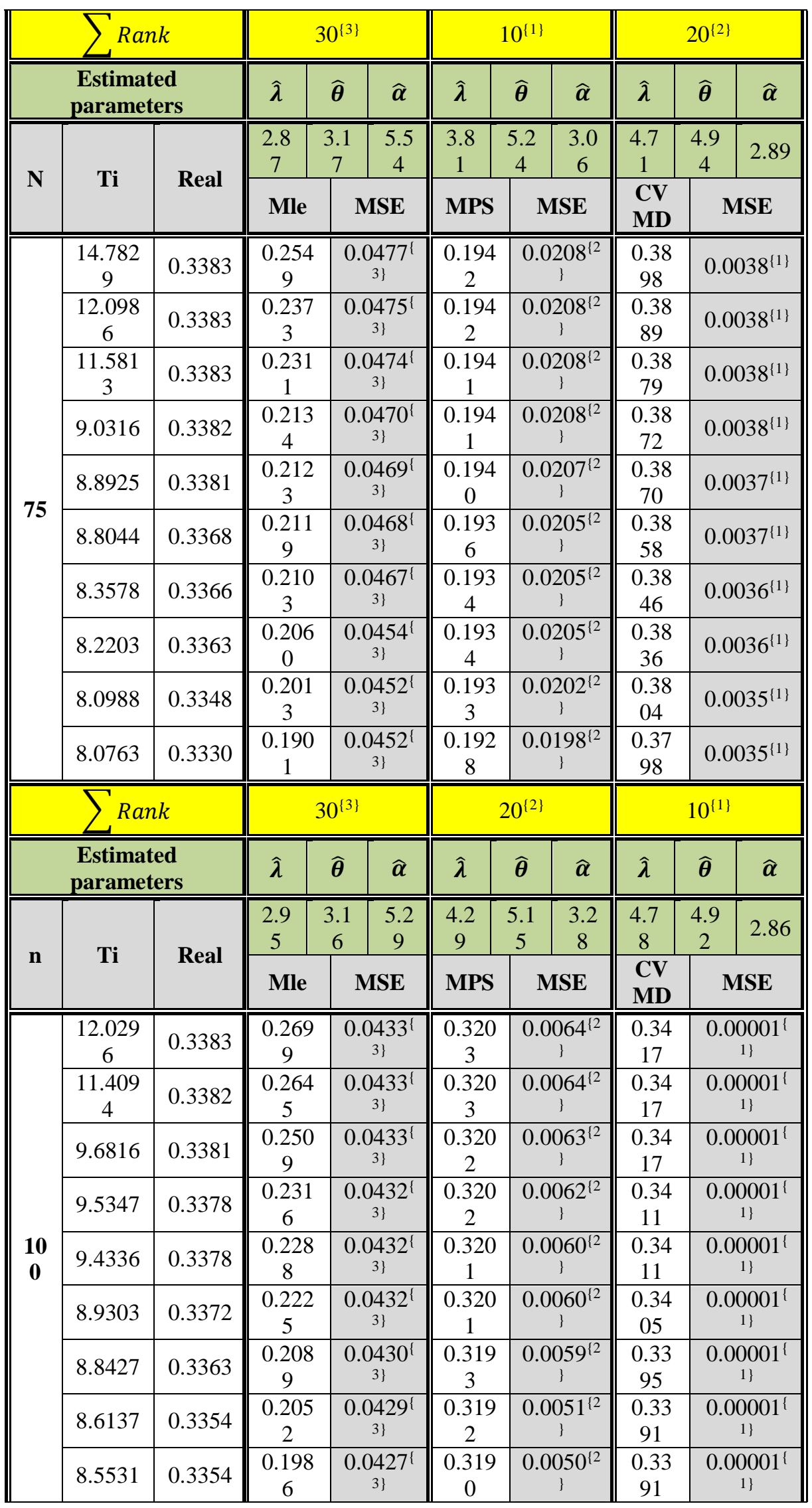




\begin{tabular}{||c|c|c||c|c||c|c||c|c||}
\hline & 8.0285 & 0.3350 & $\begin{array}{c}0.196 \\
6\end{array}$ & $\begin{array}{c}0.0427^{\{} \\
3\}\end{array}$ & $\begin{array}{c}0.318 \\
7\end{array}$ & $\begin{array}{c}0.0048^{\{2} \\
\}\end{array}$ & $\begin{array}{c}0.33 \\
88\end{array}$ & $\begin{array}{c}0.00001^{\{} \\
1\}\end{array}$ \\
\hline \hline$\sum$ Rank & \multicolumn{2}{|c|}{$30^{\{3\}}$} & \multicolumn{2}{|c|}{$20^{\{2\}}$} & \multicolumn{2}{|c|}{$10^{\{1\}}$} \\
\hline
\end{tabular}

Table (8): Simulation results for $(\lambda=2.5, \theta=5, \alpha=3.5)$

\begin{tabular}{|c|c|c|c|c|c|c|c|c|c|c|c|}
\hline \multicolumn{3}{|c|}{$\begin{array}{c}\text { Estimated } \\
\text { parameters }\end{array}$} & $\hat{\lambda}$ & $\widehat{\boldsymbol{\theta}}$ & $\widehat{\alpha}$ & $\hat{\lambda}$ & $\widehat{\boldsymbol{\theta}}$ & $\widehat{\alpha}$ & $\hat{\lambda}$ & $\widehat{\boldsymbol{\theta}}$ & $\widehat{\alpha}$ \\
\hline \multirow{2}{*}{$\mathbf{N}$} & \multirow{2}{*}{$\mathbf{T i}$} & \multirow{2}{*}{ Real } & 2.21 & 3.08 & 6.53 & 3.30 & \multicolumn{2}{|l|}{5.09} & $\begin{array}{c}2.8 \\
4\end{array}$ & $\begin{array}{c}4.7 \\
2\end{array}$ & $\begin{array}{c}3.2 \\
0\end{array}$ \\
\hline & & & Mle & \multicolumn{2}{|c|}{ MSE } & MPS & \multicolumn{2}{|c|}{ MSE } & $\begin{array}{c}\text { CVM } \\
\text { D }\end{array}$ & \multicolumn{2}{|c|}{ MSE } \\
\hline \multirow{10}{*}{35} & $\begin{array}{c}15.534 \\
5\end{array}$ & $\begin{array}{c}0.194 \\
1\end{array}$ & $\begin{array}{c}0.276 \\
8\end{array}$ & \multicolumn{2}{|c|}{$0.0522^{\{3\}}$} & 0.3519 & \multicolumn{2}{|c|}{$0.0249^{\{2\}}$} & $\begin{array}{c}0.248 \\
3\end{array}$ & \multicolumn{2}{|c|}{$\begin{array}{c}0.0039\{ \\
1\}\end{array}$} \\
\hline & $\begin{array}{c}15.427 \\
1\end{array}$ & $\begin{array}{c}0.193 \\
8\end{array}$ & $\begin{array}{c}0.241 \\
2\end{array}$ & \multicolumn{2}{|c|}{$0.0180^{\{2\}}$} & 0.3460 & \multicolumn{2}{|c|}{$0.0232^{\{3\}}$} & $\begin{array}{c}0.245 \\
1\end{array}$ & \multicolumn{2}{|c|}{$\begin{array}{c}0.0039 \\
1\}\end{array}$} \\
\hline & $\begin{array}{c}12.969 \\
2\end{array}$ & $\begin{array}{c}0.190 \\
1\end{array}$ & $\begin{array}{c}0.235 \\
2 \\
\end{array}$ & \multicolumn{2}{|c|}{$0.0143^{\{2\}}$} & 0.3372 & \multicolumn{2}{|c|}{$0.0224^{\{3\}}$} & $\begin{array}{c}0.244 \\
3\end{array}$ & \multicolumn{2}{|c|}{$\begin{array}{c}0.0039 \\
1\}\end{array}$} \\
\hline & $\begin{array}{c}12.476 \\
1\end{array}$ & $\begin{array}{c}0.187 \\
6\end{array}$ & $\begin{array}{c}0.219 \\
3\end{array}$ & \multicolumn{2}{|c|}{$0.0070^{\{2\}}$} & 0.3233 & \multicolumn{2}{|c|}{$0.0181^{\{3\}}$} & $\begin{array}{c}0.242 \\
7\end{array}$ & \multicolumn{2}{|c|}{$\begin{array}{c}0.0037^{\{} \\
1\}\end{array}$} \\
\hline & $\begin{array}{c}11.460 \\
3\end{array}$ & $\begin{array}{c}0.185 \\
7\end{array}$ & $\begin{array}{c}0.205 \\
6\end{array}$ & \multicolumn{2}{|c|}{$0.0069^{\{2\}}$} & 0.3164 & \multicolumn{2}{|c|}{$0.0177^{\{3\}}$} & $\begin{array}{c}0.240 \\
8\end{array}$ & \multicolumn{2}{|c|}{$\begin{array}{c}0.0035^{1} \\
1\}\end{array}$} \\
\hline & 9.0421 & $\begin{array}{c}0.182 \\
5\end{array}$ & $\begin{array}{c}0.191 \\
2\end{array}$ & \multicolumn{2}{|c|}{$0.0069^{\{2\}}$} & 0.3096 & & $68^{\{3\}}$ & $\begin{array}{c}0.229 \\
4\end{array}$ & & $\begin{array}{l}028^{\{} \\
1\}\end{array}$ \\
\hline & 8.9282 & $\begin{array}{c}0.181 \\
8\end{array}$ & $\begin{array}{c}0.175 \\
2\end{array}$ & 0.0 & $9^{\{2\}}$ & 0.3018 & & $35^{\{3\}}$ & $\begin{array}{c}0.228 \\
2\end{array}$ & & $\begin{array}{l}026^{1} \\
1\}\end{array}$ \\
\hline & 8.5483 & $\begin{array}{c}0.181 \\
0\end{array}$ & $\begin{array}{c}0.172 \\
5\end{array}$ & 0.0 & $9^{\{2\}}$ & 0.2883 & & $12^{\{3\}}$ & $\begin{array}{c}0.217 \\
4\end{array}$ & & $02^{\{1}$ \\
\hline & 7.6380 & $\begin{array}{c}0.180 \\
0\end{array}$ & $\begin{array}{c}0.162 \\
2\end{array}$ & 0.0 & $9^{\{2\}}$ & 0.2821 & & $02^{\{3\}}$ & $\begin{array}{c}0.211 \\
6\end{array}$ & & $\begin{array}{l}019^{\{} \\
1\}\end{array}$ \\
\hline & 6.8036 & $\begin{array}{c}0.177 \\
6 \\
\end{array}$ & $\begin{array}{c}0.136 \\
5 \\
\end{array}$ & 0.0 & $8^{\{2\}}$ & 0.2686 & & $87^{\{3\}}$ & $\begin{array}{c}0.210 \\
7\end{array}$ & & $\begin{array}{l}016^{1} \\
1\}\end{array}$ \\
\hline & $\sum R a$ & & & $21^{\{2\}}$ & & & $29^{\{3\}}$ & & & $0^{\{1\}}$ & \\
\hline & $\begin{array}{l}\text { Estimat } \\
\text { parame }\end{array}$ & & $\hat{\lambda}$ & $\widehat{\boldsymbol{\theta}}$ & $\widehat{\alpha}$ & $\hat{\lambda}$ & $\widehat{\boldsymbol{\theta}}$ & $\widehat{\alpha}$ & $\hat{\lambda}$ & $\widehat{\boldsymbol{\theta}}$ & $\widehat{\alpha}$ \\
\hline & & Do & 2.17 & 3.07 & 6.63 & 2.22 & 5.04 & 2.85 & $\begin{array}{c}2.7 \\
6\end{array}$ & $\begin{array}{c}4.7 \\
4\end{array}$ & $\begin{array}{c}3.2 \\
6\end{array}$ \\
\hline n & T1 & Real & Mle & & SE & MPS & & ISE & $\begin{array}{c}\text { CVM } \\
\text { D }\end{array}$ & & ISE \\
\hline & $\begin{array}{c}17.567 \\
3\end{array}$ & $\begin{array}{c}0.193 \\
9\end{array}$ & $\begin{array}{c}0.273 \\
4\end{array}$ & 0.0 & $30^{\{3\}}$ & 0.1894 & & $\begin{array}{l}002^{\{} \\
1\}\end{array}$ & $\begin{array}{c}0.245 \\
7\end{array}$ & & $\begin{array}{l}034^{4} \\
2\}\end{array}$ \\
\hline & $\begin{array}{c}16.027 \\
3\end{array}$ & $\begin{array}{c}0.193 \\
6\end{array}$ & $\begin{array}{c}0.260 \\
1\end{array}$ & 0.0 & $71^{\{3\}}$ & 0.1890 & & $\begin{array}{l}0002^{1} \\
1\}\end{array}$ & $\begin{array}{c}0.245 \\
5\end{array}$ & & $\begin{array}{l}034^{1} \\
2\}\end{array}$ \\
\hline & $\begin{array}{c}15.570 \\
5\end{array}$ & $\begin{array}{c}0.193 \\
4\end{array}$ & $\begin{array}{c}0.254 \\
4\end{array}$ & 0.0 & $14^{\{3\}}$ & 0.1890 & & $\begin{array}{l}0002^{\{} \\
1\}\end{array}$ & $\begin{array}{c}0.245 \\
2\end{array}$ & & $\begin{array}{l}034^{1} \\
2\}\end{array}$ \\
\hline 75 & $\begin{array}{c}12.293 \\
4\end{array}$ & $\begin{array}{c}0.193 \\
4\end{array}$ & $\begin{array}{c}0.236 \\
1\end{array}$ & 0.0 & $69^{\{3\}}$ & 0.1888 & & $\begin{array}{l}0002^{1} \\
1\}\end{array}$ & $\begin{array}{c}0.244 \\
5\end{array}$ & & $\begin{array}{l}034^{\prime} \\
2\}\end{array}$ \\
\hline & $\begin{array}{c}12.065 \\
3\end{array}$ & $\begin{array}{c}0.193 \\
1\end{array}$ & $\begin{array}{c}0.234 \\
8\end{array}$ & 0.0 & $60^{\{3\}}$ & 0.1887 & & $\begin{array}{l}0002^{1} \\
1\}\end{array}$ & $\begin{array}{c}0.242 \\
7\end{array}$ & & $\begin{array}{l}034^{1} \\
2\}\end{array}$ \\
\hline & $\begin{array}{c}11.918 \\
6\end{array}$ & $\begin{array}{c}0.193 \\
0\end{array}$ & $\begin{array}{c}0.234 \\
4\end{array}$ & 0.0 & $58^{\{3\}}$ & 0.1884 & & $\begin{array}{l}0002^{1} \\
1\}\end{array}$ & $\begin{array}{c}0.241 \\
5\end{array}$ & & $\begin{array}{l}034^{1} \\
2\}\end{array}$ \\
\hline & 11.149 & 0.191 & 0.232 & 0.0 & $46^{\{3\}}$ & 0.1864 & & $0002^{1}$ & 0.241 & & $0034^{1}$ \\
\hline
\end{tabular}


Statistical Properties \& Different Methods Of Estimation Of A New Extended Weighted Frechet Distribution.

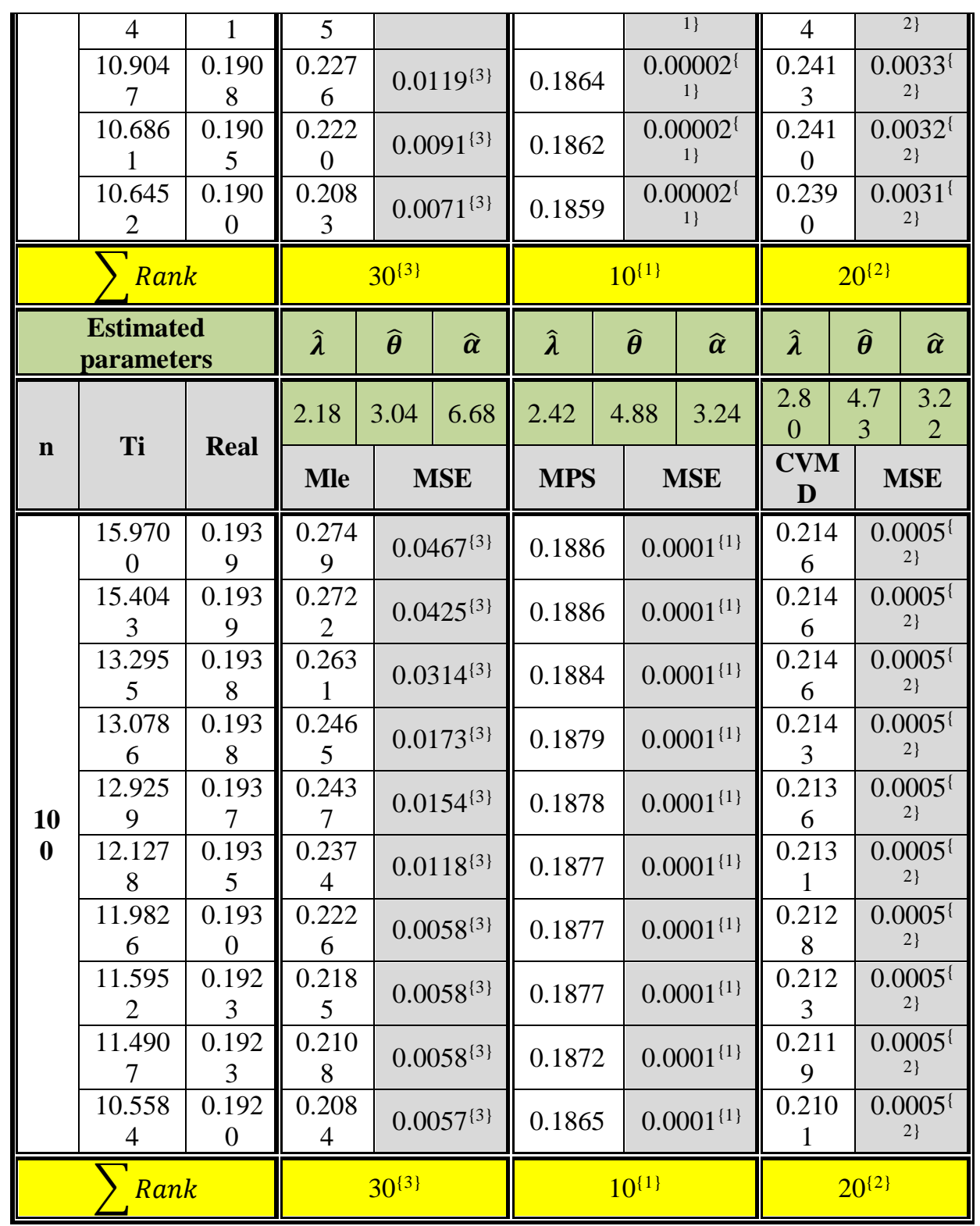

Table (9): Partial and overall ranks of all estimation methods for various combinations of $(\hat{\lambda}, \hat{\theta}, \hat{\alpha})$

\begin{tabular}{|c|c|c|c|c|}
\hline \multirow{2}{*}{$\begin{array}{c}\text { Paramerters } \\
\text { case }\end{array}$} & \multirow[t]{2}{*}{$\mathrm{N}$} & \multicolumn{3}{|c|}{ Method } \\
\hline & & MLE & PSM & $\mathrm{CVM}$ \\
\hline \multirow{3}{*}{$(\hat{\lambda}=2, \hat{\theta}=4, \hat{\alpha}=4)$} & 35 & 3 & 2 & 1 \\
\hline & 75 & 2 & 3 & 1 \\
\hline & 100 & 3 & 2 & 1 \\
\hline \multirow{3}{*}{$\lambda=3.5, \theta=4, \alpha=3.5$} & 35 & 3 & 2 & 1 \\
\hline & 75 & 3 & 1 & 2 \\
\hline & 100 & 3 & 1 & 2 \\
\hline \multirow{3}{*}{$\lambda=3.5, \theta=5, \alpha=2.5$} & 35 & 3 & 1 & 2 \\
\hline & 75 & 3 & 2 & 1 \\
\hline & 100 & 3 & 1 & 2 \\
\hline
\end{tabular}




\begin{tabular}{|c|c|c|c|c|}
\hline \multirow{3}{*}{$\lambda=4, \theta=6.5, \alpha=3$} & 35 & 3 & 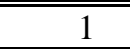 & 2 \\
\hline & 75 & 3 & 2 & 1 \\
\hline & 100 & 3 & 1 & 2 \\
\hline \multirow{3}{*}{$\lambda=3, \theta=6.5, \alpha=4$} & 35 & 3 & 2 & 1 \\
\hline & 75 & 3 & 1 & 2 \\
\hline & 100 & 3 & 2 & 1 \\
\hline \multirow{3}{*}{$\lambda=2, \theta=4, \alpha=2$} & 35 & 2.5 & 1 & 2.5 \\
\hline & 75 & 3 & 1 & 2 \\
\hline & 100 & 3 & 1 & 2 \\
\hline \multirow{3}{*}{$\lambda=4.5, \theta=5, \alpha=3$} & 35 & $\overline{3}$ & $\overline{1} 1$ & $\overline{2}$ \\
\hline & 75 & 3 & 2 & 1 \\
\hline & 100 & 3 & 2 & 1 \\
\hline \multirow{3}{*}{$\lambda=2.5, \theta=5, \alpha=3.5$} & 35 & 2 & 3 & 1 \\
\hline & 75 & 3 & 1 & 2 \\
\hline & 100 & 3 & 1 & 2 \\
\hline \multicolumn{2}{|l|}{$\sum$ Ranks } & 69.5 & $37^{37}$ & 37.5 \\
\hline \multicolumn{2}{|l|}{ Overall rank } & $\overline{3}$ & 1 & $\overline{2}$ \\
\hline \multicolumn{2}{|l|}{ Percentages } & 0.483 & 0.257 & 0.260 \\
\hline
\end{tabular}

From table (10), we can observe that:

- The maximum product spacing of the estimator method is the best method over the rest of the estimation methods.

- The sample size $(\mathrm{n}=100)$ is the best over the rest of the sample sizes.

- And the values imposed for the parameters when $(\lambda=4, \theta=6.5, \alpha=3)$.

\section{Conclusion}

In this paper, we proposed a new distribution derived from the Frechet distribution called the new extended weighted Frechet distribution. The properties of the new distribution were derived through mathematical and statistical operations. We also estimated the parameters of the new distribution by estimation methods Max. Production spacing of estimator method, and estimation of failure function by simulation.

\section{References}

1. A. Azzalini, (1985), "A class of Distribution which Includes the Normal Ones", Scandinavian Journal of Statistics, Vol.12, N.2, PP.171-178.

2. Abbas Mahdavi \& Leila Jabari, (2017), “An Extended Weighted Exponential Distribution”, Journal of Modern Applied Statistical Methods, vol.16, No.1, PP.296-307.

3. Abdulhakim A. Al-Babtain, (2020), "A new extended Rayleigh distribution", Journal of King Saud University Science, PP.2576-2581.

4. Abhimanyu Singh Yadav, et al., (2020), "Statistical properties and different methods of estimation for extended weighted inverted Rayleigh distribution", Statistics in transition, vol.21, No.2, PP.119-131.

5. Gupta, A. K., Chang, F. C. and Haung, W. J. (2002). "Some skew-symmetric models" , Random operators and Stochastic Equations, 10, PP.133-140.

6. Kamran Abbas and Tang Yincai, (2012), "Comparison of Estimation Methods for Frechet Distribution with Known Shape", Caspian Journal of Applied Sciences Research, vorl.1, No.10, PP.58-64.

7. M. K. Shakhatreh, (2012), "A two - parameter of weighted exponential distribution", Statistics and Probability Letters, PP.252-261.

8. Nadarajah, S. (2009), On the skew-logistic distributionAStA Advances in statistical Analysis, 93, PP. 187-203.

9. Rameshwar D. Gupta \& Debasis Kundu, (2009) “ A new class of weighted exponential distributions", journal of statistical computation and simulation, Vol. 43, No. 6, PP.621- 634. 